\title{
Performance of Osteoporosis Self-Assessment Tool (OST) in Predicting Osteoporosis-A Review
}

\author{
Shaanthana Subramaniam, Soelaiman Ima-Nirwana and Kok-Yong Chin * (D) \\ Department of Pharmacology, Faculty of Medicine, Universiti Kebangsaan Malaysia, Jalan Yaacob Latif, Bandar \\ Tun Razak, Cheras, Kuala Lumpur 56000, Malaysia; shaanthana_bks@hotmail.com (S.S.); \\ imasoel@ppukm.ukm.edu.my (S.I.-N.) \\ * Correspondence: chinkokyong@ppukm.ukm.edu.my; Tel.: +60-3-9145-9573
}

Received: 6 June 2018; Accepted: 4 July 2018; Published: 9 July 2018

\begin{abstract}
Bone health screening plays a vital role in the early diagnosis and treatment of osteoporosis to prevent fragility fractures among the elderly and high-risk individuals. Dual-energy X-ray absorptiometry (DXA), which detects bone mineral density, is the gold standard in diagnosing osteoporosis but is not suitable for screening. Therefore, many screening tools have been developed to identify individuals at risk for osteoporosis and prioritize them for DXA scanning. The Osteoporosis Self-assessment Tool (OST) is among the first tools established to predict osteoporosis in postmenopausal women. It can identify the population at risk for osteoporosis, but its performance varies according to ethnicity, gender, and age. Thus, these factors should be considered to ensure the optimal use of OST worldwide. Overall, OST is a simple and economical screening tool to predict osteoporosis and it can help to optimize the use of DXA.
\end{abstract}

Keywords: bone mineral density; dual-energy X-ray absorptiometry; mass screening; osteopenia; sensitivity; specificity

\section{Introduction}

Osteoporosis is a progressive bone metabolic disease. It is undetectable until a bone fracture occurs. Once osteoporosis has developed, then it is less likely to completely restore the bone strength of the patients [1]. The prevalence of osteoporosis is increasing as the global population ages rapidly. In Asia, the number of osteoporotic hip fractures is expected to rise from 1,124,060 in 2018 to 2,563,488 in 2050 [2]. The Asians also encompassed 55\% of the population at risk for fragility fractures worldwide [3]. The escalating morbidity and mortality rates due to osteoporotic fractures have distressed the patients, families, and society [4]. In addition, fragility fractures also contribute to a tremendous healthcare and economic burden. A recent meta-analysis of studies in Asia indicated that the median medical cost for hip fracture was USD 2943, representing around 19\% of the gross domestic product of the countries studied in 2014 [5]. Thus, it is crucial to identify individuals at risk for osteoporosis to enable early intervention for fracture prevention.

Dual-energy X-ray absorptiometry (DXA) is the gold standard technique used to detect osteoporosis. According to the World Health Organization (WHO), a bone mineral density $(B M D) \leq-2.5$ standard deviations $(\mathrm{SD})$ below the young adult mean (or a T-score $\leq-2.5$ ) indicates osteoporosis, while a T-score value at any site between $\leq-1.0$ and $>-2.5$ indicates a low bone mass or osteopenia [6]. Dual-energy X-ray absorptiometry cannot be widely used for osteoporosis screening due to its high cost and limited availability in most developing countries [7]. Quantitative ultrasound (QUS) has been developed as an alternative to DXA for osteoporosis screening [7]. Although QUS is portable and more economical than DXA, it may be unavailable in all primary medical settings. 
Various clinical risk assessment tools have been developed to evaluate the risk of osteoporosis [8]. These screening tools help physicians to prioritize high-risk patients for a DXA scan. Some of the screening algorithms are the Fracture Risk Assessment Tool (FRAX), Qfracture algorithm, and Garvan Fracture Risk Calculator (Garvan) [9]. The Osteoporosis Self-assessment Tool (OST) is another predictive algorithm currently in use to predict the risk for osteoporosis [10]. It was first established by Koh et al. (2001) using data of postmenopausal women from eight Asian countries. The screening algorithm was only based on age (years) and body weight $(\mathrm{kg})$ : OSTA score $=($ body weight - age) $\times 0.2$, with three osteoporosis risk categories: low risk (>-1), moderate risk ( -1 to -4$)$, and high risk $(<-4)$. It performed well to determine women at risk of osteoporosis [11]. The performance of OST among Asian men was first assessed by Kung et al. (2004) and it demonstrated a moderate performance in predicting osteoporosis [12]. OST has been known as OSTA (OST for Asians) when it is applied to Asian women. The establishment of OSTA only involved postmenopausal women and men from East and Southeast Asia. A recent article by Chin (2017) reviewed the performance of OSTA among various Asian populations, but the performance of OST in non-Asian countries was not examined [13]. Thus, the present review summarized and compared the performance of OST in determining osteoporosis risk among the Asian and non-Asian population.

\section{Literature Search}

A literature search was performed from 15 January 2018 to 4 April 2018 using two databases: PubMed and Scopus. Only original articles written in English were included in this review. The search term used was "osteoporosis self-assessment tool". The search revealed 84 articles from PubMed and 65 articles from Scopus, which resulted in a total of 149 articles. After removing 16 duplicated articles, 133 articles were screened based on title and abstract. Only studies investigating the performance of OST against DXA were considered. The present review included 44 relevant articles (Figure 1).

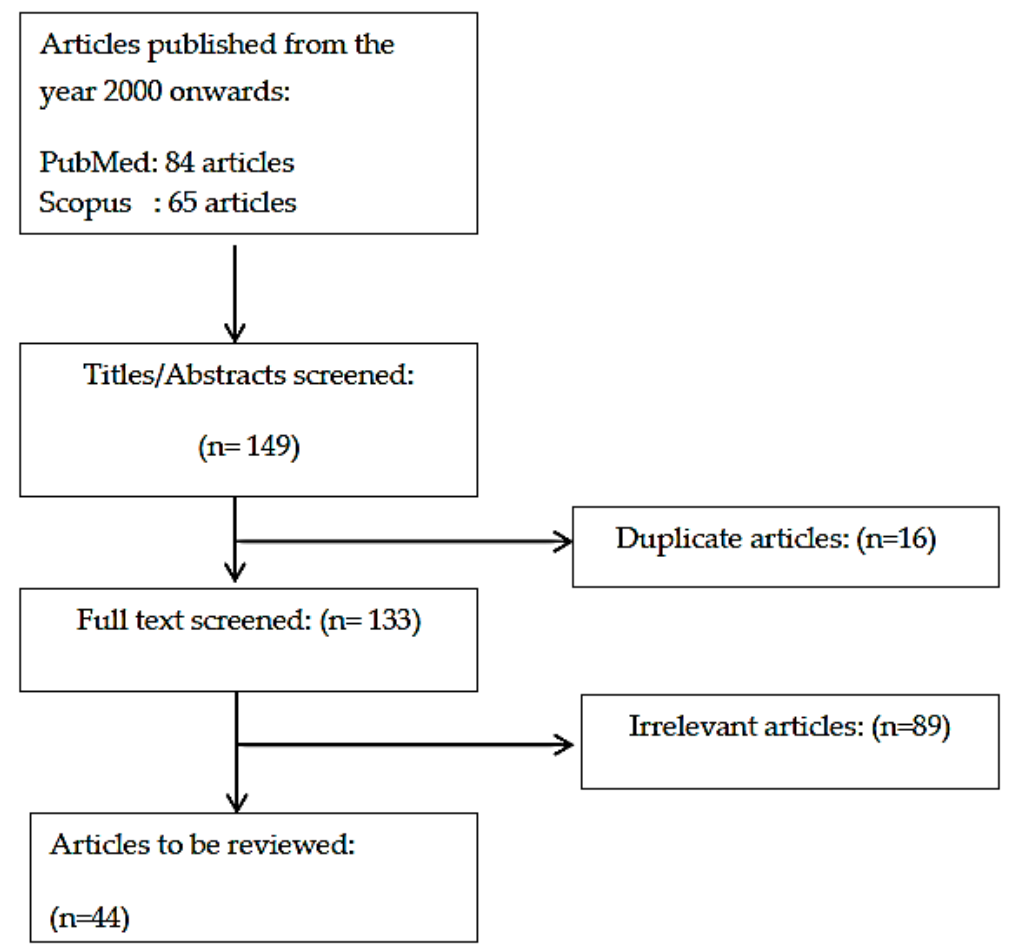

Figure 1. Flowchart of literature search. 


\section{Performance of OST among Asians}

\subsection{Performance of OST among Asian Women}

The osteoporosis self-assessment tool for Asians (OSTA) was first developed by Koh et al. (2001) using data of postmenopausal women from eight Asian countries. The final algorithm only selected age and body weight as the predictors, creating the formula: OSTA score = (body weight in $\mathrm{kg}-\mathrm{age}$ in years $) \times 0.2$. Based on the truncated product of this formula, the women could be divided into three risk categories: low-risk $(>-1)$, moderate-risk $(-1$ to -4$)$, and high-risk $(<-4)$. The predictive values of these scores were good, as indicated by the fact that $61 \%$ women categorized as high-risk were osteoporotic compared to 3\% in the low-risk group in their study [11]. Its performance (cutoff $=-1$; sensitivity $=91 \%$; specificity $=45 \%$; AUC $=0.79$ ) was superior than the reported values of the SOFSURF index [14], Osteoporosis Risk Assessment Index (ORAI), and Simple Calculated Osteoporosis Risk Estimation (SCORE) [15]. The performance of OSTA was subsequently validated in other East Asian populations, such as Chinese [16] and Korean women [17], and comparable results were obtained. This is not surprising, considering that a majority of the subjects in the development phase were East Asians [11]. In addition, the validation study by Huang et al. (2015) showed that OSTA performed better when BMD at the femoral neck was used as the reference, and when the women tested were older [16]. The site difference is probably due to the presence of osteophyte at the lumbar spine, which distorts its BMD. This finding was validated in similar studies conducted among Thai women $[18,19]$. The age difference coincides with the development population, which was elderly women (mean age: $62.3 \pm 6.2$ years) [11]. The limitation of OSTA in predicting osteoporosis among younger women was also observed in Thai women [19].

\subsection{Performance of OST among Asian Men}

Men also suffer from osteoporosis and their post-fracture mortality rate is higher than women [20]. Therefore, OSTA was developed for men by Kung et al. in 2004 based on data of community-dwelling Chinese men (age range: 50-93 years) in Hong Kong. The algorithm and cutoff values were the same as reported by Koh et al. (2001), but its performance in the development and validation cohort was not as good as in postmenopausal women (cutoff $=-1$; sensitivity $=71-73 \%$, specificity $=68 \%$; AUC $=0.780-0.790$ ) [12]. OSTA was subsequently validated in the Chinese Han population [21] and Korean men [22], and both studies obtained a sensitivity $>80 \%$. In contrast, OSTA (cutoff $<-1$ ) demonstrated a low sensitivity (27.6-28.5\%) and a high specificity $(89.2-92.7 \%)$ in a large study involving Chinese men of a wide age range (40-96 years) [23]. Sub-analysis revealed that similar to women, OSTA only performed well among older subjects [23].

\subsection{Performance of OST with Modified Cutoff Values}

Since the original cutoff values for OSTA were established in postmenopausal elderly women, predominantly Eastern Asians, its performance, in terms of sensitivity and specificity, may vary according to sex, age, and ethnic groups. Hence, modification to the cutoff values may be necessary to ensure the optimal performance of OSTA. This hypothesis was tested by Bhat et al. (2016) among Indian subjects (aged $>50$ years) using a cutoff of 2 and OSTA achieved high sensitivity (sensitivity $=95.7 \%$, specificity $=33.6 \%$, AUC $=0.702$ ) in predicting osteoporosis among the subjects [24]. However, OSTA failed to show similar performance in other populations (Chinese men with the cutoff -3.5 , sensitivity $=47.3 \%$ [4]; Taiwanese men with the cutoff -1.86 , sensitivity $69.2 \%$ [25]). In the subsequent discussion, the readers should notice the changes in cutoff values in many studies.

\subsection{Performance of OST in Comparison with Other Screening Tools}

QUS is another popular osteoporosis screening tool [7]. It was reported to have a strong association with BMD and bone mineral content (BMC) measured by DXA [26]. In three studies, OSTA was found to perform equally with QUS $[8,27,28]$. Thus, OSTA can be used in medical settings without QUS. 
However, it should be noted that OSTA and QUS cannot be used interchangeably because the two tools are not equivalent [29].

OSTA was also compared against other screening algorithms. With a modified cutoff of -2 , OSTA showed a sensitivity of $90.0-91.9 \%$ among postmenopausal women, which was better than SCORE (cutoff $\geq 8$ ), ORAI (cutoff $\geq 20$ ), ABONE (cutoff $=3$ ), and WEIGHT (cutoff $<54 \mathrm{~kg}$ ) [30]. In another study among the Taiwanese elderly, OSTA achieved a sensitivity of $100 \%$ in men and women, outperforming ABONE (cutoff $\geq 2$ ), BWC $(<70 \mathrm{~kg}$ ), FRAX and GARVAN (cutoff $>3 \%$ for hip fracture, $>20 \%$ for major osteoporotic fracture), ORAI (cutoff $\geq 9$ ), OSIRIS (cutoff $\leq 1$ ), OSTA (cutoff $<-1$ ), and SCORE (cutoff $\geq 6$ ) [27].

The universality of OSTA was challenged by some researchers with osteoporosis screening algorithms designed for the local populations. The performance of OSTA was proven to be equivalent in some cases. For example, the Khon Kaen Osteoporosis Study (KKOS) (cutoff $<-1$ ) scoring system shared a similar performance with OSTA (cutoff $<-1$ ) in Thai women (AUC: 0.64 vs. 0.65) [31]. The Beijing Friendship Hospital Osteoporosis Self-Assessment Tool (BFH-OST) also performed similarly to OSTA (cutoff $=-1$ ) in predicting osteoporosis in Chinese Han women and men (AUC: $0.795-0.797$ vs. 0.732-0.782), despite having a higher sensitivity $(73.58-89.92 \%$ vs. $50.42-65.28 \%$ ) [32,33]. Among the South Indian elderly, the performance of the Male Osteoporosis Risk Estimation Score (MORES) (cutoff $=6$ ) was also equivalent to OSTA (cutoff $\leq 2)$ (AUC: 0.760 vs. 0.778) [34].

In some cases, the local algorithms were better than OSTA in osteoporosis screening. Among Taiwanese women, the Osteoporosis Preclinical Assessment Tool (OPAT) containing four predictors (age, menopausal status, weight, and alkaline phosphatase activity) performed better than OSTA (sensitivity: $87 \%$ vs. $78 \%$; AUC: 0.77 vs. 0.69) [35]. Similarly, the Korean Osteoporosis Risk-Assessment Model (KORAM) (cutoff < -9) also performed better than OSTA in predicting osteoporosis among Korean menopausal women (cutoff < 0) (AUC: 0.682-0.709 vs. 0.617-0.626) [36]. The Malaysian Osteoporosis Screening Tool (MOST) (cutoff $\geq 4$ ) was also superior to OSTA (cutoff $<2$ ) (AUC: 67.6\% vs. $52 \%$ ) in predicting osteoporosis among healthy women [37]. Despite that, these local screening tools might not be useful outside the local settings.

A summary of the literature on the performance of OST among Asians is listed in Table 1. 
Table 1. Performance of OST among Asians.

\begin{tabular}{|c|c|c|c|c|c|c|c|c|c|}
\hline Study & Objective & Subject Description & $\begin{array}{c}\text { Number of } \\
\text { Subjects Recruited }\end{array}$ & Methods & Cutoff & Sensitivity (\%) & Specificity $(\%)$ & AUC & Remarks \\
\hline \multirow{4}{*}{$\begin{array}{l}\text { Koh et al. (2001) } \\
\text { [11] }\end{array}$} & \multirow{4}{*}{$\begin{array}{l}\text { To develop Osteoporosis } \\
\text { Screening Tool for } \\
\text { Asians (OSTA) }\end{array}$} & \multirow{4}{*}{$\begin{array}{l}\text { Postmenopausal women (mean age } \\
62 \text { years) recruited from } 2 \text { clinics in } \\
\text { eight Asia countries. }\end{array}$} & \multirow{4}{*}{860} & \multirow{4}{*}{$\begin{array}{c}\text { DXA: } \\
\text { 8 Hologic machines (3 Model 4500, } 5 \\
\text { Model 2000), } \\
\text { 4 Norland (2 XR-36, } 1 \text { S-26, } 1 \text { XR-26), } \\
\text { 12 Lunar (3 DPX-IQ. 6 DPX-L, 3 } \\
\text { Expert) machines }\end{array}$} & $\begin{array}{c}\text { OSTA }<-1 \\
\text { T-score }<-2.5\end{array}$ & 91 & 45 & 0.79 & \\
\hline & & & & & $\begin{array}{l}\text { SOFSURF }<1.4 \\
\text { T-score }<-2.5\end{array}$ & 90 & 46 & 0.77 & \\
\hline & & & & & $\begin{array}{c}\text { ORAI }<15 \\
\text { T-score }<-2.5\end{array}$ & 84 & 52 & 0.76 & \\
\hline & & & & & $\begin{array}{c}\text { SCORE }<10 \\
\text { T-score }<-2.5\end{array}$ & 90 & 33 & 0.77 & \\
\hline \multirow{2}{*}{$\begin{array}{l}\text { Park et al. (2003) } \\
\quad[17]\end{array}$} & \multirow{2}{*}{$\begin{array}{l}\text { To validate the effectiveness } \\
\text { of OSTA in identifying } \\
\text { osteoporosis among Korean } \\
\text { women }\end{array}$} & \multirow{2}{*}{$\begin{array}{l}\text { Postmenopausal women from a } \\
\text { clinic in Korea and who were not on } \\
\text { hormone replacement therapy } \\
\text { (mean age: } 59.1 \pm 7.7 \text { years) }\end{array}$} & \multirow{2}{*}{1101} & \multirow{2}{*}{$\begin{array}{c}\text { DXA } \\
\text { GE Lunar model DPQ-IQ } \\
\text { BMD at FN }\end{array}$} & $\begin{aligned} & \text { OSTA }<-1 \\
& \text { T-score } \leq-2.0\end{aligned}$ & 80 & 72 & 0.85 & \multirow{2}{*}{ Single-centered } \\
\hline & & & & & $\begin{array}{c}\text { OSTA }<-1 \\
\text { T-score } \leq-2.5\end{array}$ & 87 & 67 & 0.873 & \\
\hline \multirow{4}{*}{$\begin{array}{c}\text { Geater et al. (2004) } \\
\text { [18] }\end{array}$} & \multirow{4}{*}{$\begin{array}{l}\text { To validate the performance } \\
\text { of OSTA in predicting } \\
\text { osteoporosis among Korean } \\
\text { women }\end{array}$} & \multirow{4}{*}{$\begin{array}{l}\text { Thai post-menopausal women } \\
\text { (mean age: } 60.5 \pm 9.7 \text { years) without } \\
\text { risk of osteoporosis }\end{array}$} & \multirow{4}{*}{388} & \multirow{4}{*}{$\begin{array}{c}\text { DXA } \\
\text { Lunar, Madison } \\
\text { BMD at FN and LS }\end{array}$} & $\begin{array}{c}\text { OSTA }<-1 \\
\text { FN T-score }<-2.5\end{array}$ & 93.5 & 60.8 & $\begin{array}{c}\text { Value not } \\
\text { mentioned }\end{array}$ & \\
\hline & & & & & $\begin{array}{c}\text { OSTA }<-1 \\
\text { LS T-score }<-2.5\end{array}$ & 79.5 & 69.5 & $\begin{array}{c}\text { Value not } \\
\text { mentioned }\end{array}$ & \\
\hline & & & & & $\begin{array}{c}\text { OSTA }<0 \\
\text { FN T-score }<-2.5\end{array}$ & 93.5 & 29.8 & $\begin{array}{l}\text { Value not } \\
\text { mentioned }\end{array}$ & \\
\hline & & & & & $\begin{array}{c}\text { OSTA }<0 \\
\text { LS T-score }<-2.5\end{array}$ & 92.4 & 35.7 & $\begin{array}{l}\text { Value not } \\
\text { mentioned }\end{array}$ & \\
\hline \multirow{4}{*}{$\begin{array}{c}\text { Huang et al. (2015) } \\
\text { [16] }\end{array}$} & \multirow{4}{*}{$\begin{array}{l}\text { To determine the } \\
\text { performance of OSTA among } \\
\text { middle-aged and old women }\end{array}$} & \multirow{4}{*}{$\begin{array}{l}\text { Healthy women (age range: } 40-96 \\
\text { years) from a hospital in Chengdu } \\
\text { region, China }\end{array}$} & \multirow{4}{*}{15,752} & \multirow{4}{*}{$\begin{array}{l}\text { DXA (Lunar Prodigy- GE } \\
\text { Healthcare, Madison, WI, USA) } \\
\text { BMD at LS, FN, and TH }\end{array}$} & $\begin{array}{c}\text { OSTA }<-1 \\
\text { LS T-score }<-1\end{array}$ & 56.9 & 87.7 & 0.812 & \\
\hline & & & & & $\begin{array}{c}\text { OSTA }<-1 \\
\text { LS T-score }<-2.5\end{array}$ & 77.3 & 73.5 & 0.812 & \\
\hline & & & & & $\begin{array}{c}\text { OSTA }<-1 \\
\text { FN T-score }<-1\end{array}$ & 56.2 & 89.8 & 0.822 & \\
\hline & & & & & $\begin{array}{c}\text { OSTA }<-1 \\
\text { FN T-score }<-2.5\end{array}$ & 88.1 & 69.3 & 0.822 & \\
\hline \multirow{3}{*}{$\begin{array}{l}\text { Yang et al. (2015) } \\
\text { [21] }\end{array}$} & \multirow{3}{*}{$\begin{array}{l}\text { To validate OSTA among } \\
\text { elderly males to determine } \\
\text { the risk of primary } \\
\text { osteoporosis }\end{array}$} & \multirow{3}{*}{$\begin{array}{l}\text { Healthy males (mean age: } \\
65.17 \pm 9.29 \text { years) }\end{array}$} & \multirow{3}{*}{245} & \multirow{3}{*}{$\begin{array}{l}\text { DXA (Hologic, Inc., Bedford, } \\
\text { MA, USA) } \\
\text { BMD at LS and LF }\end{array}$} & $\begin{array}{c}\text { OSTA }<1 \\
\text { FN T-score }<-2.5 \\
\end{array}$ & 84 & 49 & 0.712 & \\
\hline & & & & & $\begin{array}{c}\text { OSTA }<1 \\
\text { TH T-score }<-2.5\end{array}$ & Value not stated & Value not stated & 0.658 & \\
\hline & & & & & $\begin{array}{c}\text { OSTA }<1 \\
\text { LS T-score }<-2.5\end{array}$ & Value not stated & Value not stated & 0.535 & \\
\hline
\end{tabular}


Table 1. Cont

\begin{tabular}{|c|c|c|c|c|c|c|c|c|c|}
\hline Study & Objective & Subject Description & $\begin{array}{c}\text { Number of } \\
\text { Subjects Recruited }\end{array}$ & Methods & Cutoff & Sensitivity (\%) & Specificity (\%) & AUC & Remarks \\
\hline \multirow{4}{*}{$\begin{array}{l}\text { Oh et al. (2016) } \\
\text { [22] }\end{array}$} & \multirow{4}{*}{$\begin{array}{l}\text { To compare the effectiveness } \\
\text { of Korean Osteoporosis } \\
\text { Risk-Assessment Model for } \\
\text { Men (KORAM-M) and OSTA }\end{array}$} & \multirow{4}{*}{$\begin{array}{l}\text { Men aged } 50 \text { and above from } 2009 \\
\text { and } 2010 \text { Korean National Health } \\
\text { and Nutrition Examination Survey }\end{array}$} & \multirow{4}{*}{$\begin{array}{c}\text { Development } \\
\text { phase: } 1340 \\
\text { Validation phase: } \\
1110\end{array}$} & \multirow{4}{*}{$\begin{array}{c}\text { DXA } \\
\text { Hologic Discovery } \\
\text { BMD at FN or LS }\end{array}$} & $\begin{array}{l}\text { Development: } \\
\text { OSTA }<-1\end{array}$ & 90.8 & 36.9 & 0.639 & \\
\hline & & & & & KORAM-M $<-9$ & 90.8 & 42.4 & 0.666 & \\
\hline & & & & & $\begin{array}{l}\text { Validation: } \\
\text { OSTA }<-1\end{array}$ & 92.3 & 33.2 & 0.627 & \\
\hline & & & & & KORAM-M $<-9$ & 87.9 & 39.7 & 0.638 & \\
\hline \multirow{4}{*}{$\begin{array}{l}\text { Huang et al. (2017) } \\
\text { [23] }\end{array}$} & \multirow{4}{*}{$\begin{array}{l}\text { To assess the effectiveness of } \\
\text { OSTA using various cutoffs }\end{array}$} & \multirow{4}{*}{$\begin{array}{l}\text { Healthy men aged } 40-96 \text { years } \\
\text { recruited from a hospital in } \\
\text { Chengdu region, China }\end{array}$} & \multirow{4}{*}{11,039} & \multirow{4}{*}{$\begin{array}{l}\text { DXA (GE Lunar, Madison, WI, USA) } \\
\text { BMD at LS and FN }\end{array}$} & $\begin{array}{c}\text { OSTA }<-1 \\
\text { LS T-score }<-1 \\
\end{array}$ & 27.6 & 89.2 & \multirow{4}{*}{$\begin{array}{l}\text { value not } \\
\text { stated }\end{array}$} & \\
\hline & & & & & $\begin{array}{c}\text { OSTA }<-1 \\
\text { LS T-score } \leq-2.5\end{array}$ & 57.3 & 86.7 & & \\
\hline & & & & & $\begin{array}{c}\text { OSTA }<-1 \\
\text { FN T-score }<-1\end{array}$ & 28.5 & 92.7 & & \\
\hline & & & & & $\begin{array}{c}\text { OSTA }<-1 \\
\text { FN T-score } \leq-2.5\end{array}$ & 65.9 & 87.0 & & \\
\hline $\begin{array}{l}\text { Bhat et al. (2017) } \\
\text { [24] }\end{array}$ & $\begin{array}{l}\text { To evaluate the performance } \\
\text { of OSTA in predicting OP } \\
\text { among Indian men }\end{array}$ & $\begin{array}{l}\text { Indian men above } 50 \text { years and } \\
\text { without apparent risk of OP }\end{array}$ & 257 & $\begin{array}{l}\text { DXA (QDR } 4500 \mathrm{~A} \text {, Hologic Inc., } \\
\text { Bedford, MA, USA) } \\
\text { BMD at LS, TH and FN }\end{array}$ & $\begin{array}{c}\text { OSTA } \leq 2 \\
\text { T-score at any sites } \\
\leq-2.5\end{array}$ & 95.7 & 33.6 & 0.702 & \\
\hline \multirow{8}{*}{$\begin{array}{l}\text { Zha et al. (2014) } \\
\text { [4] }\end{array}$} & \multirow{8}{*}{$\begin{array}{l}\text { To validate OSTA and QUS } \\
\text { and their combination in } \\
\text { predicting OP among the } \\
\text { high-risk population }\end{array}$} & \multirow{8}{*}{ Chinese men (mean age: 78.0 years) } & \multirow{8}{*}{472} & \multirow{8}{*}{$\begin{array}{l}\text { DXA (Discovery A, Hologic, USA) } \\
\text { QUS (Sahara clinical bone } \\
\text { sonometer- Hologic) } \\
\text { BMD at LS and LH }\end{array}$} & $\begin{array}{c}\text { OSTA }<-3.5 \\
\text { FN T-score }<-2.5\end{array}$ & 65.5 & 74.8 & 0.724 & \multirow{8}{*}{$\begin{array}{l}\text { Small sample } \\
\text { size } \\
\text { Sample } \\
\text { recruited from } \\
\text { a single centre }\end{array}$} \\
\hline & & & & & $\begin{array}{c}\text { OSTA }<-3.5 \\
\text { TH T-score }<-2.5\end{array}$ & 81.8 & 72.7 & 0.787 & \\
\hline & & & & & $\begin{array}{c}\text { OSTA }<-3.5 \\
\text { LS T-score }<-2.5\end{array}$ & 45.4 & 74.7 & 0.652 & \\
\hline & & & & & $\begin{array}{c}\text { OSTA }<-3.5 \\
\text { T-score at any site }< \\
-2.5 \\
\end{array}$ & 47.3 & 76.8 & 0.676 & \\
\hline & & & & & $\begin{array}{c}\text { QUS }<-1.15 \\
\text { FN T-score }<-2.5\end{array}$ & 88.9 & 47.4 & 0.762 & \\
\hline & & & & & $\begin{array}{c}\text { QUS }<-2.15 \\
\text { TH T-score }<-2.5\end{array}$ & 82.4 & 86.6 & 0.883 & \\
\hline & & & & & $\begin{array}{c}\text { QUS }<-1.25 \\
\text { LS T-score }<-2.5 \\
\end{array}$ & 82.7 & 57.9 & 0.750 & \\
\hline & & & & & $\begin{array}{c}\text { QUS }<-1.25 \\
\text { T-score at any site }< \\
-2.5\end{array}$ & 80.4 & 59.7 & 0.762 & \\
\hline
\end{tabular}


Table 1. Cont

\begin{tabular}{|c|c|c|c|c|c|c|c|c|c|}
\hline \multirow{3}{*}{$\begin{array}{l}\text { Chang \& Yang } \\
\text { (2016) } \\
\text { [25] }\end{array}$} & \multirow{3}{*}{$\begin{array}{l}\text { To conduct a cutoff study } \\
\text { among males by using OST, } \\
\text { BMI, age and body weight }\end{array}$} & \multirow{3}{*}{$\begin{array}{l}\text { Retrospective data of Northern } \\
\text { Taiwan males with mean age of } \\
71.9 \pm 13.3 \text { years }\end{array}$} & \multirow{3}{*}{834} & \multirow{3}{*}{$\begin{array}{l}\text { DXA } \\
\text { BMD at FN }\end{array}$} & $\begin{array}{c}\text { OST }<-1.86 \\
\text { T-score } \leq-2.5\end{array}$ & 69.2 & 63 & 0.70 & \multirow{3}{*}{$\begin{array}{l}\text { Subjects were } \\
\text { patients } \\
\text { referred to } \\
\text { BMD test by } \\
\text { orthopaedic } \\
\text { surgeons }\end{array}$} \\
\hline & & & & & $\begin{array}{l}\text { BMI }<23 \mathrm{~kg} / \mathrm{m}^{2} \\
\text { T-score } \leq-2.5\end{array}$ & 60.4 & 61.6 & 0.63 & \\
\hline & & & & & $\begin{array}{l}\text { Weight }<58.8 \mathrm{~kg} \\
\text { T-score } \leq-2.5\end{array}$ & 43.9 & 78.2 & 0.66 & \\
\hline \multirow{4}{*}{$\begin{array}{l}\text { Kung et al. (2003) } \\
{[28]}\end{array}$} & \multirow{4}{*}{$\begin{array}{l}\text { To develop OSTA for Asian } \\
\text { men }\end{array}$} & \multirow{4}{*}{$\begin{array}{l}\text { Community-dwelling Chinese men } \\
\text { (age: 50-93 years) }\end{array}$} & \multirow{4}{*}{420} & \multirow{4}{*}{$\begin{array}{c}\text { Development followed by validation } \\
\text { in } 356 \text { men } \\
\text { DXA: QDR } 2000 \text { Plus Hologic, } \\
\text { Waltham, MA, USA } \\
\text { BMD at LS and LF } \\
\text { QUS: Sahara Hologic, Waltham, } \\
\text { MA, USA }\end{array}$} & $\begin{array}{c}\text { Development: } \\
\text { OSTA }<-1 \\
\text { T-score } \leq-2.5 \\
\end{array}$ & 73 & 68 & 0.790 & \multirow{4}{*}{$\begin{array}{l}\text { Subjects were } \\
\text { not selected } \\
\text { randomly }\end{array}$} \\
\hline & & & & & $\begin{array}{c}\text { Validation: } \\
\text { OSTA }<-1 \\
\text { T-score } \leq-2.5 \\
\end{array}$ & 71 & 68 & 0.780 & \\
\hline & & & & & $\begin{array}{c}\text { Validation: } \\
\text { QUI }<-1.2 \\
\text { T-score } \leq-2.5\end{array}$ & 76 & 72 & 0.80 & \\
\hline & & & & & $\begin{array}{c}\text { Either OSTA }<-1 \text { or } \mathrm{QUI}<-2.5 \\
\text { T-score } \leq-2.5\end{array}$ & 88 & 64 & 0.82 & \\
\hline \multirow{10}{*}{$\begin{array}{c}\text { Chan et al. (2006) } \\
\text { [30] }\end{array}$} & \multirow{10}{*}{$\begin{array}{l}\text { To compare the validity of } \\
\text { various OP risk indices in } \\
\text { elderly Chinese females }\end{array}$} & \multirow{10}{*}{$\begin{array}{c}\text { Community-dwelling } \\
\text { postmenopausal women (age } \geq 55 \text { ) }\end{array}$} & \multirow{10}{*}{135} & \multirow{10}{*}{$\begin{array}{l}\text { DXA (Hologic QDR } 4500 \text { W) } \\
\text { BMD at FN and LS }\end{array}$} & OSTA (cutoff $\leq-2$ & 90.9 & 58.8 & 0.82 & \multirow{10}{*}{$\begin{array}{l}\text { Small sample } \\
\text { size }\end{array}$} \\
\hline & & & & & $\begin{array}{l}\text { FN I-score } \leq-2.5 \\
\text { LS T-score }<-2.5\end{array}$ & 91.9 & 42.9 & 0.73 & \\
\hline & & & & & SCORE (cutoff $\geq 8$ ) & 93.9 & 60.8 & 0.80 & \\
\hline & & & & & $\begin{array}{l}\text { FN 1-score } \leq-2.5 \\
\text { LS T-score }<-2.5\end{array}$ & 86.5 & 60.2 & 0.72 & \\
\hline & & & & & ORAI (cutoff $\geq 20$ ) & 75.8 & 66.7 & 0.76 & \\
\hline & & & & & $\begin{array}{l}\text { FN I-score } \leq-2.5 \\
\text { LS T-score }<-2.5\end{array}$ & 62 & 62 & 0.68 & \\
\hline & & & & & ABONE (cutoff $=3$ ) & 81.8 & 55.9 & 0.70 & \\
\hline & & & & & $\begin{array}{l}\text { FN I-score } \leq-2.5 \\
\text { LS T-score } \leq-2.5\end{array}$ & 73 & 54.1 & 0.66 & \\
\hline & & & & & SCORE (cutoff $\geq 8$ ) & 67.9 & 77.5 & 0.78 & \\
\hline & & & & & FN T-score $\leq$ & 62.2 & 76.5 & 0.73 & \\
\hline \multirow{6}{*}{$\begin{array}{l}\text { Chaovisitsaree et al. } \\
\text { (2007) } \\
{[19]}\end{array}$} & \multirow{6}{*}{$\begin{array}{l}\text { To compare OSTA with DXA } \\
\text { in determining osteopenia } \\
\text { and osteoporosis } \\
\text { menopausal women }\end{array}$} & \multirow{6}{*}{$\begin{array}{l}\text { Thai menopausal women (age range: } \\
45-87 \text { years) from Menopause Clinic } \\
\text { in Chiang Mai University }\end{array}$} & \multirow{6}{*}{315} & \multirow{6}{*}{$\begin{array}{c}\text { DXA } \\
\text { BMD at FN, LS and radius }\end{array}$} & OSTA $<-1$ & 36.2 & 71.4 & \multirow{6}{*}{$\begin{array}{l}\text { Value not } \\
\text { mentioned }\end{array}$} & \\
\hline & & & & & $\begin{array}{l}\text { LS T-score } \leq-1 \\
\text { FN T-score } \leq-1\end{array}$ & 40.6 & 72.0 & & \\
\hline & & & & & Radius T-score $\leq-1$ & 48.3 & 75.1 & & \\
\hline & & & & & OSTA $<-1$ & 45.8 & 68.9 & & \\
\hline & & & & & $\begin{array}{l}\text { LS I-score } \leq-2.5 \\
\text { FN T-score }<-2.5\end{array}$ & 75.0 & 67.8 & & \\
\hline & & & & & Radius T-score $\leq-2.5$ & 60 & 68.5 & & \\
\hline
\end{tabular}


Table 1. Cont.

\begin{tabular}{|c|c|c|c|c|c|c|c|c|c|}
\hline \multirow{11}{*}{$\begin{array}{l}\text { Chen et al. (2016) } \\
\text { [27] }\end{array}$} & \multirow{11}{*}{$\begin{array}{l}\text { To compare the performance } \\
\text { of different screening tools to } \\
\text { predict fracture or OP risk } \\
\text { among older poople }\end{array}$} & \multirow{11}{*}{$\begin{array}{l}\text { Community-dwelling older people } \\
\text { aged } 60 \text { and above (mean age: } 67.4 \\
\pm 6,4 \text { years) recruited from Tanzi } \\
\text { District, Taiwan }\end{array}$} & \multirow{11}{*}{553} & \multirow{11}{*}{$\begin{array}{c}\text { DXA } \\
\text { Hologic Discovery Wi Bone } \\
\text { Densitometer } \\
\text { BMD at FN } \\
\text { QUS } \\
\text { GE Lunar, Madison, WI }\end{array}$} & $\begin{array}{c}\text { QUS } \\
\text { FN T-score } \leq-2.5\end{array}$ & $\begin{array}{l}20(\mathrm{M}) \\
59(\mathrm{~F})\end{array}$ & $\begin{array}{l}86(\mathrm{M}) \\
75(\mathrm{~F})\end{array}$ & $\begin{array}{l}0.72(\mathrm{M}) \\
0.77(\mathrm{~F})\end{array}$ & \\
\hline & & & & & $\mathrm{ABONE} \geq 2$ & $\begin{array}{l}100(\mathrm{M}) \\
100(\mathrm{~F})\end{array}$ & $\begin{array}{l}28(\mathrm{M}) \\
10(\mathrm{~F})\end{array}$ & $\begin{array}{l}0.78(\mathrm{M}) \\
0.70(\mathrm{~F})\end{array}$ & \\
\hline & & & & & BWC $<70 \mathrm{~kg}$ & $\begin{array}{l}100(\mathrm{M}) \\
100(\mathrm{~F})\end{array}$ & $\begin{array}{c}36(\mathrm{M}) \\
7(\mathrm{~F})\end{array}$ & $\begin{array}{l}0.92(\mathrm{M}) \\
0.80(\mathrm{~F})\end{array}$ & \\
\hline & & & & & $\begin{array}{c}\text { FRAX } \\
\text { Hip fracture }(>3 \%)\end{array}$ & $\begin{array}{l}80(\mathrm{M}) \\
83(\mathrm{~F})\end{array}$ & $\begin{array}{l}71(\mathrm{M}) \\
54(\mathrm{~F})\end{array}$ & $\begin{array}{l}0.86(\mathrm{M}) \\
0.75(\mathrm{~F})\end{array}$ & \\
\hline & & & & & MOF $(>20 \%)$ & $\begin{array}{l}0(\mathrm{M}) \\
17(\mathrm{~F}) \\
\end{array}$ & $\begin{array}{l}99(\mathrm{M}) \\
96(\mathrm{~F})\end{array}$ & $\begin{array}{l}0.77(\mathrm{M}) \\
0.71(\mathrm{~F})\end{array}$ & \\
\hline & & & & & $\begin{array}{c}\text { GARVAN } \\
\text { Hip fracture }(>3 \%)\end{array}$ & $\begin{array}{l}60(\mathrm{M}) \\
28(\mathrm{~F})\end{array}$ & $\begin{array}{l}79(\mathrm{M}) \\
95(\mathrm{~F})\end{array}$ & $\begin{array}{l}0.72(\mathrm{M}) \\
0.80(\mathrm{~F})\end{array}$ & \\
\hline & & & & & Any osteoporotic fracture $(>20 \%)$ & $\begin{array}{l}20(\mathrm{M}) \\
55(\mathrm{~F})\end{array}$ & $\begin{array}{l}96(\mathrm{M}) \\
73(\mathrm{~F})\end{array}$ & $\begin{array}{l}0.72(\mathrm{M}) \\
0.75(\mathrm{~F})\end{array}$ & \\
\hline & & & & & ORAI $\geq 9$ & $\begin{array}{l}100(\mathrm{M}) \\
100(\mathrm{~F})\end{array}$ & $\begin{array}{c}19(\mathrm{M}) \\
5(\mathrm{~F})\end{array}$ & $\begin{array}{l}0.87(\mathrm{M}) \\
0.77(\mathrm{~F})\end{array}$ & \\
\hline & & & & & OSIRIS $\leq 1$ & $\begin{array}{l}100(\mathrm{M}) \\
100(\mathrm{~F})\end{array}$ & $\begin{array}{c}29(\mathrm{M}) \\
6(\mathrm{~F})\end{array}$ & $\begin{array}{l}0.94(\mathrm{M}) \\
0.83(\mathrm{~F})\end{array}$ & \\
\hline & & & & & $\mathrm{OSTA} \leq-1$ & $\begin{array}{l}100(\mathrm{M}) \\
100(\mathrm{~F})\end{array}$ & $\begin{array}{l}58(\mathrm{M}) \\
27(\mathrm{~F})\end{array}$ & $\begin{array}{l}0.94(\mathrm{M}) \\
0.83(\mathrm{~F})\end{array}$ & \\
\hline & & & & & SCORE $\geq 6$ & $\begin{array}{l}100(\mathrm{M}) \\
100(\mathrm{~F})\end{array}$ & $\begin{array}{l}45(\mathrm{M}) \\
15(\mathrm{~F})\end{array}$ & $\begin{array}{l}0.91(\mathrm{M}) \\
0.80(\mathrm{~F})\end{array}$ & \\
\hline \multirow{2}{*}{$\begin{array}{l}\text { Chen et al. (2017) } \\
\text { [35] }\end{array}$} & \multirow{2}{*}{$\begin{array}{c}\text { To establish a prediction } \\
\text { model to identify osteopenia } \\
\text { risk in women aged } \\
40-55 \text { years }\end{array}$} & \multirow{2}{*}{$\begin{array}{l}\text { Taiwanese women recruited from a } \\
\text { health checkup centre }\end{array}$} & \multirow{2}{*}{1350} & \multirow{2}{*}{$\begin{array}{c}\text { DXA (DPX-L; GE Lunar Health Care, } \\
\text { Madison, WI, USA) } \\
\text { BMD at LS }\end{array}$} & OSTA $\leq 1$ & 78 & 47 & 0.69 & \multirow{2}{*}{$\begin{array}{l}\text { Novel algorithm to } \\
\text { predict osteopenia }\end{array}$} \\
\hline & & & & & $\begin{array}{c}\text { OPAT } \geq 1 \\
-1 \geq \text { T-score }>-2.5 \text { at LS }\end{array}$ & 87 & 42 & 0.77 & \\
\hline \multirow{4}{*}{$\begin{array}{c}\text { Panichyawat \& } \\
\text { Tanmahasamut } \\
\text { (2012) } \\
\text { [31] }\end{array}$} & \multirow{4}{*}{$\begin{array}{l}\text { To compare the performance } \\
\text { of OSTA and Khon Kaen } \\
\text { Osteoporosis Study (KKOS) } \\
\text { scoring system to predict OP } \\
\text { among postmenopausal } \\
\text { women in Thailand }\end{array}$} & \multirow{4}{*}{$\begin{array}{l}\text { Postmenopausal women (mean age: } \\
55.8 \pm 5.9 \text { years) from menopause } \\
\text { clinic }\end{array}$} & \multirow{4}{*}{441} & \multirow{4}{*}{$\begin{array}{c}\text { DXA } \\
\text { BMD at FN and TH }\end{array}$} & $\begin{array}{c}\text { OSTA }=-1 \\
\text { T-score at any site } \leq-2.5\end{array}$ & 51.7 & 77.4 & 0.65 & \multirow{4}{*}{$\begin{array}{l}\text { Subjects from a } \\
\text { single centre }\end{array}$} \\
\hline & & & & & $\begin{array}{c}\text { OSTA }=0 \\
\text { T-score at any site } \leq-2.5\end{array}$ & 66.7 & 57.1 & 0.62 & \\
\hline & & & & & $\begin{array}{c}\text { KKOS }=-1 \\
\text { T-score at any site } \leq-2.5\end{array}$ & 56.3 & 71.8 & 0.64 & \\
\hline & & & & & $\begin{array}{c}\text { KKOS }=0 \\
\text { T-score at any site } \leq-2.5\end{array}$ & 57.5 & 67.2 & 0.62 & \\
\hline
\end{tabular}


Table 1. Cont.

\begin{tabular}{|c|c|c|c|c|c|c|c|c|}
\hline \multirow{8}{*}{$\begin{array}{l}\text { Oh et al. (2013) } \\
\text { [36] }\end{array}$} & \multirow{8}{*}{$\begin{array}{l}\text { To develop Korean } \\
\text { Osteoporosis } \\
\text { Risk-Assessment Model } \\
\text { (KORAM) and compare its } \\
\text { performance with OSTA }\end{array}$} & \multirow{8}{*}{$\begin{array}{l}\text { Postmenopausal women who } \\
\text { participated in the } 2009 \text { and } 2010 \\
\text { Korean National Health and } \\
\text { Nutrition Examination Survey }\end{array}$} & \multirow{8}{*}{$\begin{array}{l}\text { Development: } \\
1209 \\
\text { Validation: } \\
1046\end{array}$} & \multirow{8}{*}{$\begin{array}{c}\text { DXA } \\
\text { QDR Discovery, Hologic } \\
\text { BMD at TF, FN and LS }\end{array}$} & $\begin{array}{c}\text { Development: } \\
\text { OSTA < } \\
\text { FN or LS T-score < } \\
-2.5\end{array}$ & 96.8 & 28.3 & 0.626 \\
\hline & & & & & $\begin{array}{c}\text { OSTA }<0 \\
\text { FN or LS T-score }< \\
-2.0\end{array}$ & 93.7 & 34.6 & 0.641 \\
\hline & & & & & $\begin{array}{c}\text { KORAM }<-9 \\
\text { FN or LS T-score }< \\
-2.5\end{array}$ & 91.2 & 50.6 & 0.709 \\
\hline & & & & & $\begin{array}{c}\text { KORAM }<-9 \\
\text { FN or LS T-score }< \\
-2.0 \\
\end{array}$ & 85.2 & 60.1 & 0.726 \\
\hline & & & & & $\begin{array}{c}\text { Validation: } \\
\text { OSTA <0 } \\
\text { FN or LS T-score }< \\
-2.5\end{array}$ & 94.2 & 29.2 & 0.617 \\
\hline & & & & & $\begin{array}{c}\text { OSTA }<0 \\
\text { FN or LS T-score }< \\
-2.0 \\
\end{array}$ & 90.9 & 35.0 & 0.629 \\
\hline & & & & & $\begin{array}{c}\text { KORAM }<-9 \\
\text { FN or LS T-score }< \\
-2.5\end{array}$ & 84.8 & 51.6 & 0.682 \\
\hline & & & & & $\begin{array}{c}\text { KORAM }<-9 \\
\text { FN or LS T-score }< \\
-2.0\end{array}$ & 79.2 & 60.2 & 0.697 \\
\hline \multirow{5}{*}{$\begin{array}{l}\text { Lim et al. (2011) } \\
{[\text { [37] }}\end{array}$} & \multirow{5}{*}{$\begin{array}{l}\text { To develop and validate } \\
\text { Malaysian Osteoporosis } \\
\text { Screening Tool (MOST) to } \\
\text { detect low BMD in Malaysia }\end{array}$} & \multirow{5}{*}{$\begin{array}{l}\text { Healthy women (mean age: } 51.3 \pm \\
5.4 \text { years) from a residential area }\end{array}$} & \multirow{5}{*}{$\begin{array}{l}\text { Development: } \\
514 \\
\text { Validation: } \\
72\end{array}$} & \multirow{5}{*}{$\begin{array}{c}\text { DXA } \\
\text { Norland XR-36 } \\
\text { BMD at FN and LS }\end{array}$} & $\begin{array}{c}\text { OST }<2 \\
\text { FN T-score } \leq-2.5\end{array}$ & 88 & 52 & \multirow{5}{*}{$\begin{array}{l}\text { Value not } \\
\text { mentioned }\end{array}$} \\
\hline & & & & & $\begin{array}{c}\text { ORAI }>8 \\
\text { FN T-score } \leq-2.5\end{array}$ & 90 & 52 & \\
\hline & & & & & $\begin{array}{c}\text { SCORE }>7 \\
\text { FN T-score } \leq-2.5 \\
\end{array}$ & 89 & 58 & \\
\hline & & & & & $\begin{array}{c}\text { SOFSURF }>-1 \\
\text { FN T-score } \leq-2.5\end{array}$ & 92 & 37 & \\
\hline & & & & & $\begin{array}{c}\text { MOST } \geq 4 \\
\text { FN/LS T-score } \\
\leq-2.5\end{array}$ & $\begin{array}{l}\text { Development: } \\
\quad 80.2 \\
\text { Validation: } \\
100\end{array}$ & $\begin{array}{l}\text { Development: } \\
\quad 55.5 \\
\text { Validation: } \\
67.6\end{array}$ & \\
\hline
\end{tabular}


Table 1. Cont.

\begin{tabular}{|c|c|c|c|c|c|c|c|c|c|}
\hline \multirow{2}{*}{$\begin{array}{l}\text { Ma et al. (2016) } \\
\text { [33] }\end{array}$} & \multirow{2}{*}{$\begin{array}{l}\text { To compare the performance } \\
\text { of OSTA and BFH in } \\
\text { determining osteoporosis } \\
\text { among postmenopausal Han } \\
\text { Chinese women }\end{array}$} & \multirow{2}{*}{$\begin{array}{l}\text { Community-dwelling Han Chinese } \\
\text { postmenopausal women with age } \\
\text { range of } 40-89 \text { years (man age: } \\
60.71 \pm 8.47 \text { years) }\end{array}$} & \multirow{2}{*}{1721} & \multirow{2}{*}{$\begin{array}{c}\text { DXA } \\
\text { Hologic Discovery QDR Wi } \\
\text { BMD at LS, FN and TH }\end{array}$} & $\begin{array}{c}\text { OSTA }<-1 \\
\text { T-score at any sites } \\
<-2.5 \\
\end{array}$ & 65.28 & 77.15 & 0.782 & \multirow{2}{*}{$\begin{array}{l}\text { Subjects from } \\
\text { a single centre }\end{array}$} \\
\hline & & & & & $\begin{array}{c}\text { BFH-OST }<-9.1 \\
\text { T-score at any sites } \\
<-2.5\end{array}$ & 73.58 & 72.66 & 0.797 & \\
\hline \multirow{3}{*}{$\begin{array}{l}\text { Lin et al. (2017) } \\
\text { [32] }\end{array}$} & \multirow{3}{*}{$\begin{array}{l}\text { To assess the performance } \\
\text { new screening tool to } \\
\text { determine osteoporosis }\end{array}$} & \multirow{3}{*}{$\begin{array}{c}\text { Development phase: } \\
\text { Community-dwelling Han Chinese } \\
\text { males aged } 50 \text { and above (mean age: } \\
65.42 \pm 8.8 \text { ) } \\
\text { Validation phase: } \\
\text { Hospital-dwelling Han Chinese men }\end{array}$} & \multirow{3}{*}{$\begin{array}{c}\text { Development: } \\
1870 \\
\text { Validation: } \\
574\end{array}$} & \multirow{3}{*}{$\begin{array}{c}\text { DXA } \\
\text { Discovery Wi, QDR series, Hologic, } \\
\text { Waltham, MA, USA } \\
\text { BMD at hip and LS }\end{array}$} & $\begin{array}{c}\text { Development: } \\
\text { BFH-OSTM } \leq 70 \\
\text { T-score }<-2.5\end{array}$ & 84.96 & 53.49 & 0.763 & \\
\hline & & & & & $\begin{array}{c}\text { Validation: } \\
\text { OSTA } \leq-1 \\
\text { T-score }<-2.5\end{array}$ & 50.42 & 82.20 & 0.732 & \\
\hline & & & & & $\begin{array}{c}\text { BFH-OSTM } \leq 70 \\
\text { T-score }<-2.5\end{array}$ & 89.92 & 48.57 & 0.795 & \\
\hline \multirow{4}{*}{$\begin{array}{l}\text { Satyaraddi et al. } \\
\qquad(2017) \\
{[34]}\end{array}$} & \multirow{4}{*}{$\begin{array}{l}\text { To evaluate the performance } \\
\text { of OSTA and Male } \\
\text { Osteoporosis Risk Estimation } \\
\text { Score (MORES) in predicting } \\
\text { OP among } \\
\text { South Indian rural elderly } \\
\text { men }\end{array}$} & \multirow{4}{*}{$\begin{array}{l}\text { Indian men aged } 65 \text { and above } \\
\text { (mean age: } 71.9 \pm 5.2 \text { years) } \\
\text { recruited by cluster random } \\
\text { sampling }\end{array}$} & \multirow{4}{*}{512} & \multirow{4}{*}{$\begin{array}{c}\text { DXA } \\
\text { Hologic QDR4500 Discovery A } \\
\text { BMD at LS and FN }\end{array}$} & $\begin{array}{c}\text { OSTA } \leq 2 \\
\text { LS T-score } \leq-2.5\end{array}$ & 94 & 17 & 0.716 & \multirow{4}{*}{$\begin{array}{l}\text { Further } \\
\text { validation } \\
\text { study is } \\
\text { needed for a } \\
\text { larger cohort } \\
\text { of subjects }\end{array}$} \\
\hline & & & & & FN T-score $\leq-2.5$ & 99 & 18 & 0.778 & \\
\hline & & & & & $\begin{array}{c}\text { MORES } \geq 6 \\
\text { LS T-score } \leq-2.5\end{array}$ & 98 & 15 & 0.855 & \\
\hline & & & & & FN T-score $\leq-2.5$ & 98 & 13 & 0.760 & \\
\hline
\end{tabular}

Abbreviation: AP, anteroposterior; AUC, area under curve; BMD, bone mineral density; BWC, body weight criteria; LS, lumbar spine; FN, femoral neck; TH, total hip; MOF,

Major osteoporotic fracture; $\mathrm{OP}$, osteoporosis; $\mathrm{PF}$, proximal femur. 


\section{Performance of OST among Non-Asians}

\subsection{Performance of OST among Non-Asian Women}

Although developed for Asians, the performance of OST has also been validated in non-Asians. The algorithm is the same as reported by Koh et al. (2001), but the cutoff values have been optimized to suit the designated populations. The performance of OST (cutoff $<2$ ) was good in determining Caucasian women at risk of osteoporosis (sensitivity $=86-95.3 \%$; specificity $=39.6-40 \%$; AUC $=0.726-0.82)[10,38]$. A study indicated that the performance of OST was similar between younger (cutoff $\leq 1$ for $45-64$ years) and older women (and cutoff $\leq-1$ for $>65$ years) when different cutoff values were used [39].

\subsection{Performance of OST among Non-Asian Men}

The use of OST in predicting bone health among non-Asian men was also validated. In Caucasian men, OST was able to predict individuals with osteoporosis (sensitivity $=93 \%$, specificity $=66 \%$, $\mathrm{AUC}=0.836$ ) [40]. The performance was better when BMD at total hip (sensitivity $=87.5 \%$; specificity $=58.2 \%$; AUC $=0.787$ ) was used as the reference compared to the lumbar spine (sensitivity $=63.6 \%$; specificity $=59.5 \%$; AUC $=0.66)$ [41]. When operated in younger men $(\geq 35$ years), OST performed optimally when the cutoff was modified to $<4$ (sensitivity $=83 \%$, specificity $=57 \%$, AUC $=0.83$ ) [42] .

\subsection{Performance of OST with Modified Cutoff Values}

The cutoff values of OST need to be optimized in different non-Asian populations. In comparison to the original -1 for Asians, a cutoff $\leq 2$ was tested for Caucasian to obtain a similar sensitivity value [43]. Other cutoff values, such as $<3$ for Portuguese men (sensitivity $=75.5 \%$, specificity $=50.0 \%$, AUC $=0.632$ ) [44], < 6 for veteran US men (sensitivity $=82.6 \%$, specificity $=33.6 \%$, AUC $=0.67$ ) [45], and $<4$ for African men (sensitivity $=83 \%$, specificity $=57 \%$, AUC 0.83) [42], have been adopted previously. The non-Asians' cutoff values are generally higher than Asians' because they have a higher body weight.

Even within the same ethnicity, the cutoff values of OST need to be modified based on sex. A Spanish study showed that OST performed optimally at a cutoff of 2 in women (sensitivity =94\%, specificity $=59 \%, \mathrm{AUC}=0.762$ ) and 3 in men (sensitivity $=39 \%$, specificity $=86 \%, \mathrm{AUC}=0.623$ ) [46] .

In a group of men with rheumatoid arthritis, OST was weak (sensitivity $=64 \%$; specificity $=54 \%$ ) in determining those with low BMD even though the cutoff was modified to $<4$. The researchers indicated that the performance of OST could be limited by the low lean body mass of patients with rheumatoid arthritis [47].

\subsection{Performance of OST in Comparison with Other Screening Tools}

In comparison with other screening algorithms, OSTA (at various optimized cutoff values) performed similarly to ABONE [48], SCORE [10,48-53], SOFSURF [49,51], ORAI [10,48,49,51,52], OSIRIS [10,49], United States Preventive Services Task Force (USPSTF)-FRAX [52-54], RF [52], BMI [52], pBW [49], Weight Criterion [48], and QUS [49] in women. It also performed similarly to the male-specific screening tool, Mscore, developed by Zimering et al. (2007) [55]. Only the study of Hawker et al. (2012) reported that OST was weak (sensitivity $=47 \%$, AUC $=0.69$ ) in identifying women with low BMD when compared to a new screening tool developed by the study (sensitivity $=93 \%$, AUC $=0.75$ ) [56].

A summary of the literature on the performance of OST among non-Asians is listed in Table 2. 
Table 2. Performance of OST for non-Asians

\begin{tabular}{|c|c|c|c|c|c|c|c|c|c|}
\hline Study & Objective & Subject Description & $\begin{array}{c}\text { Number of Subjects } \\
\text { Recruited }\end{array}$ & Methods & Cutoff & Sensitivity (\%) & Specificity (\%) & AUC & Remarks \\
\hline \multirow{4}{*}{$\begin{array}{c}\text { Richy et al. } 2004 \\
\text { [10] }\end{array}$} & \multirow{4}{*}{$\begin{array}{l}\text { To validate and compare } \\
\text { the performance of OST } \\
\text { with other osteoporosis } \\
\text { risk indices }\end{array}$} & \multirow{4}{*}{$\begin{array}{l}\text { Postmenopausal White } \\
\text { women (mean age: } 61.5 \pm 8.8 \\
\text { years) without Paget's } \\
\text { disease or advanced } \\
\text { osteoarthritis }\end{array}$} & \multirow{4}{*}{4035} & \multirow{4}{*}{$\begin{array}{l}\text { DXA: Hologic QDR } 2000 \\
\text { BMD at any site }\end{array}$} & $\begin{array}{c}\text { OST }<2 \\
\text { T-score } \leq-2.5 \\
\text { T-score } \leq-2 \\
\end{array}$ & $\begin{array}{l}86 \\
82\end{array}$ & $\begin{array}{l}40 \\
44\end{array}$ & $\begin{array}{l}0.726 \\
0.713\end{array}$ & \multirow{4}{*}{$\begin{array}{l}\text { Subjects were either } \\
\text { referred or came } \\
\text { spontaneously for } \\
\text { osteoporosis evaluation } \\
\text { and may differ in some } \\
\text { ways from the general } \\
\text { population }\end{array}$} \\
\hline & & & & & $\begin{array}{c}\text { SCORE }>7 \\
\text { T-score } \leq-2.5 \\
\text { T-score } \leq-2\end{array}$ & $\begin{array}{l}86 \\
78\end{array}$ & $\begin{array}{l}40 \\
46\end{array}$ & $\begin{array}{l}0.708 \\
0.700\end{array}$ & \\
\hline & & & & & $\begin{array}{c}\text { ORAI }>8 \\
\text { T-score } \leq-2.5 \\
\text { T-score } \leq-2\end{array}$ & $\begin{array}{l}76 \\
73\end{array}$ & $\begin{array}{l}48 \\
51\end{array}$ & $\begin{array}{l}0.670 \\
0.668\end{array}$ & \\
\hline & & & & & $\begin{array}{c}\text { OSIRIS }<1 \\
\text { T-score } \leq-2.5 \\
\text { T-score } \leq-2 \\
\end{array}$ & $\begin{array}{l}64 \\
58\end{array}$ & $\begin{array}{l}69 \\
73\end{array}$ & $\begin{array}{l}0.730 \\
0.717\end{array}$ & \\
\hline \multirow{4}{*}{$\begin{array}{c}\text { Cadarette et al. } 2004 \\
\text { [38] }\end{array}$} & \multirow{4}{*}{$\begin{array}{l}\text { To validate the } \\
\text { performance of } \\
\text { osteoporosis risk indices to } \\
\text { determine women at high } \\
\text { risk of osteoporosis }\end{array}$} & \multirow{4}{*}{$\begin{array}{l}\text { Women (mean age: } 62.4 \\
\text { years) with age range of } \\
45-90 \text { years }\end{array}$} & \multirow{4}{*}{644} & \multirow{4}{*}{$\begin{array}{c}\text { DXA } \\
\text { BMD at FN and LS }\end{array}$} & $\begin{array}{c}\text { ORAI }>8 \\
\text { T-score }<-2.5\end{array}$ & 92.5 & 38.7 & 0.80 & \multirow{4}{*}{$\begin{array}{c}\text { The study included } \\
\text { data from women who } \\
\text { have been selected for } \\
\text { BMD testing }\end{array}$} \\
\hline & & & & & $\begin{array}{c}\text { OST chart }<2 \\
\text { T-score }<-2.5\end{array}$ & 91.5 & 45.7 & 0.82 & \\
\hline & & & & & $\begin{array}{c}\text { OST equation }<2 \\
\text { T-score }<-2.5\end{array}$ & 95.3 & 39.6 & 0.82 & \\
\hline & & & & & $\begin{array}{c}\text { Body weight } \\
\text { criterion }<70 \mathrm{~kg}\end{array}$ & 93.4 & 34.6 & 0.73 & \\
\hline \multirow[t]{2}{*}{$\begin{array}{c}\text { Adler et al. } 2003 \\
\text { [40] }\end{array}$} & \multirow[t]{2}{*}{$\begin{array}{l}\text { To assess the performance } \\
\text { of OST in men }\end{array}$} & \multirow{2}{*}{$\begin{array}{l}\text { American men (mean age: } \\
64.3 \pm 12.3 \text { years) recruited } \\
\text { from pulmonary and } \\
\text { rheumatology clinic }\end{array}$} & \multirow[t]{2}{*}{181} & \multirow{2}{*}{$\begin{array}{c}\text { Hologic QDR } 4500 \\
\text { (Hologic, Inc., Bedford, } \\
\text { MA, USA) } \\
\text { BMD at LS, FN and TH }\end{array}$} & $\begin{array}{c}\text { OST }=3 \\
\text { T-score } \leq-2.5 \\
\end{array}$ & 93 & 66 & 0.836 & \multirow{2}{*}{$\begin{array}{l}\text { The study was not } \\
\text { designed specifically to } \\
\text { validate OST } \\
\text { Small sample size }\end{array}$} \\
\hline & & & & & $\begin{array}{c}\text { OST }=3 \\
\text { T-score } \leq-2.0\end{array}$ & 74 & 72 & 0.815 & \\
\hline \multirow{3}{*}{$\begin{array}{c}\text { Ghazi et al. (2007) } \\
\text { [41] }\end{array}$} & \multirow{3}{*}{$\begin{array}{l}\text { To evaluate the } \\
\text { performance of OST in } \\
\text { predicting men with low } \\
\text { BMD }\end{array}$} & \multirow{3}{*}{$\begin{array}{l}\text { White men (age range: } 50-85 \\
\text { years) from a hospital in } \\
\text { Morocco }\end{array}$} & \multirow{3}{*}{229} & \multirow{3}{*}{$\begin{array}{c}\text { DXA } \\
\text { Lunar Prodigy Vision } \\
\text { machine (GE) } \\
\text { BMD at TH and LS }\end{array}$} & $\begin{array}{c}\text { OST }=2 \\
\text { TH T-score } \leq-2.5\end{array}$ & 87.5 & 58.2 & 0.787 & \\
\hline & & & & & $\begin{array}{c}\text { OST }=2 \\
\text { LS T-score } \leq-2.5\end{array}$ & 63.6 & 59.5 & 0.660 & \\
\hline & & & & & $\begin{array}{c}\text { OST }=2 \\
\text { T-score } \leq-2.5 \text { at } \\
\text { any site }\end{array}$ & 64 & 60.3 & 0.667 & \\
\hline
\end{tabular}


Table 2. Cont.

\begin{tabular}{|c|c|c|c|c|c|c|c|c|c|}
\hline \multirow{10}{*}{$\begin{array}{c}\text { Lynn et al. (2008) } \\
\text { [43] }\end{array}$} & \multirow{10}{*}{$\begin{array}{l}\text { To evaluate the use of OST, } \\
\text { Male Osteoporosis } \\
\text { Screening Tool (MOST) and } \\
\text { Quantitative Ultrasound } \\
\text { Index (QUI) and body } \\
\text { weight as osteoporosis } \\
\text { screening tools }\end{array}$} & \multirow{10}{*}{$\begin{array}{c}\text { Caucasian and Hong Kong } \\
\text { Chinese men, aged } \geq 65 \\
\text { years and } \\
\text { community-dwelling from } \\
\text { Osteoporotic Fractures in } \\
\text { Men (MrOS) Study }\end{array}$} & \multirow{10}{*}{$\begin{array}{l}4658 \text { Caucasian men } \\
\text { 1914 Hong Kong } \\
\text { Chinese men }\end{array}$} & \multirow{10}{*}{$\begin{array}{c}\text { DXA } \\
\text { Hologic QDR 4500 W } \\
\text { (Hologic Inc.) } \\
\text { BMD at LS and PF }\end{array}$} & \multicolumn{4}{|c|}{ Caucasian: } & \\
\hline & & & & & $\begin{array}{c}\text { OST } \leq 1 \\
\text { T-score at any site } \\
\leq-2.5\end{array}$ & 79.3 & 48.5 & \multirow[t]{2}{*}{0.714} & \\
\hline & & & & & $\begin{array}{c}\text { OST } \leq 2 \\
\text { T-score at any site } \\
\leq-2.5\end{array}$ & 87.6 & 36.1 & & \\
\hline & & & & & $\begin{array}{c}\text { MOST } \leq 26 \\
\text { T-score at any site } \\
\leq-2.5\end{array}$ & 88.5 & 50 & \multirow[t]{2}{*}{0.799} & \\
\hline & & & & & $\begin{array}{c}\text { MOST } \leq 27 \\
\text { T-score at any site } \\
\leq-2.5\end{array}$ & 94.7 & 37.8 & & \\
\hline & & & & & \multicolumn{4}{|c|}{ Chinese: } & \\
\hline & & & & & $\begin{array}{c}\text { OST } \leq-2 \\
\text { T-score at any site } \\
\leq-2.5\end{array}$ & 81.8 & 56.2 & \multirow[t]{2}{*}{0.759} & \\
\hline & & & & & $\begin{array}{c}\text { OST } \leq-1 \\
\text { T-score at any site } \\
\leq-2.5\end{array}$ & 91.9 & 36.4 & & \\
\hline & & & & & $\begin{array}{c}\text { MOST } \leq 21 \\
\text { T-score at any site } \\
\leq-2.5\end{array}$ & 86.8 & 59.3 & \multirow[t]{2}{*}{0.831} & \\
\hline & & & & & $\begin{array}{c}\text { MOST } \leq 22 \\
\text { T-score at any site } \\
\leq-2.5\end{array}$ & 94.2 & 42.3 & & \\
\hline \multirow{6}{*}{$\begin{array}{c}\text { Gourlay et al. (2005) } \\
\text { [39] }\end{array}$} & \multirow{6}{*}{$\begin{array}{l}\text { To compare the } \\
\text { performance of three } \\
\text { osteoporosis risk indices in } \\
\text { two different age groups. }\end{array}$} & \multirow{6}{*}{$\begin{array}{l}\text { Postmenopausal women } \\
\text { aged 45-96 years }\end{array}$} & \multirow{6}{*}{4035} & \multirow{6}{*}{$\begin{array}{c}\text { DXA: Hologic QDR 1000, } \\
2000 \text { and } 4500 \text { (Hologic } \\
\text { Inc., Waltham, MA, USA) } \\
\text { BMD at FN }\end{array}$} & $\begin{array}{c}\text { OST } \leq 1 \\
\text { Ages } 45-64 \text { years }\end{array}$ & 89.2 & 45 & 0.768 & \multirow{6}{*}{$\begin{array}{l}\text { Subjects from a single } \\
\text { centre }\end{array}$} \\
\hline & & & & & $\begin{array}{c}\text { OST } \leq-1 \\
\text { Ages } \geq 65 \text { years }\end{array}$ & 84.6 & 47.5 & 0.762 & \\
\hline & & & & & $\begin{array}{c}\text { ORAI } \geq 8 \\
\text { Ages } 45-64 \text { years }\end{array}$ & 88.5 & 46.2 & 0.750 & \\
\hline & & & & & $\begin{array}{c}\text { ORAI } \geq 13 \\
\text { Ages } \geq 65 \text { years }\end{array}$ & 89.2 & 44.7 & 0.747 & \\
\hline & & & & & $\begin{array}{c}\text { SCORE } \geq 7 \\
\text { Ages } 45-64 \text { years }\end{array}$ & 88.5 & 39.8 & 0.757 & \\
\hline & & & & & $\begin{array}{c}\text { SCORE } \geq 11 \\
\text { Ages } \geq 65 \text { years }\end{array}$ & 88.8 & 42.3 & 0.745 & \\
\hline
\end{tabular}


Table 2. Cont.

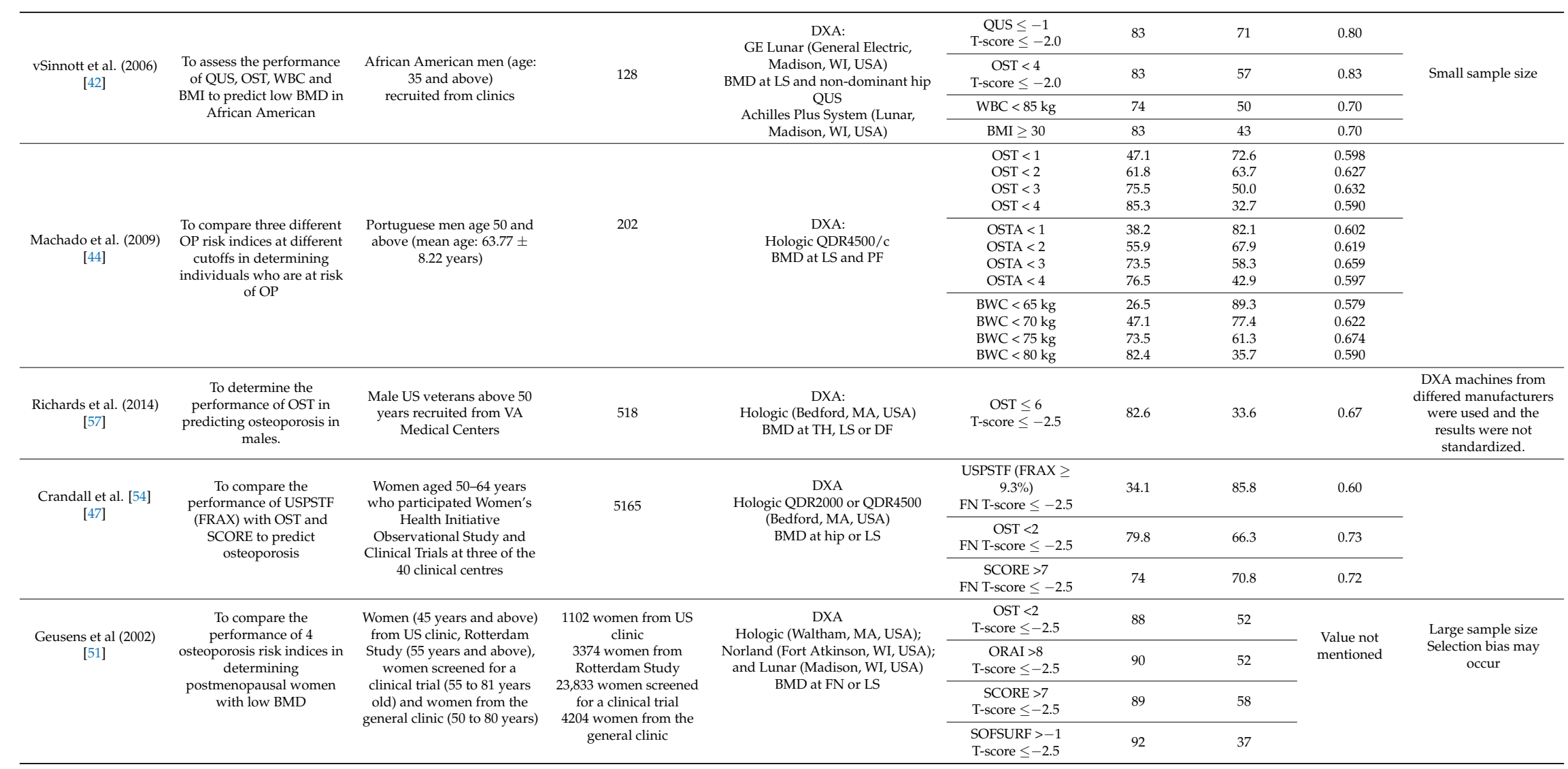


Table 2. Cont.

\begin{tabular}{|c|c|c|c|c|c|c|c|c|c|}
\hline \multirow{5}{*}{$\begin{array}{c}\text { Wallace et al. (2004) } \\
\text { [48] }\end{array}$} & \multirow{5}{*}{$\begin{array}{l}\text { To compare the } \\
\text { performance of five } \\
\text { osteoporosis risk indices in } \\
\text { determining } \\
\text { postmenopausal } \\
\text { African-American women } \\
\text { with low BMD }\end{array}$} & \multirow{5}{*}{$\begin{array}{l}\text { Women (mean age: } 59.4 \pm \\
12.5 \text { years) from an } \\
\text { osteoporosis study }\end{array}$} & \multirow{5}{*}{174} & \multirow{5}{*}{$\begin{array}{c}\text { DXA } \\
\text { Hologic QDR } 2000 \\
\text { BMD at FN }\end{array}$} & $\begin{array}{c}\text { ABONE } \geq 2 \\
\text { T-score } \leq-2.5\end{array}$ & 73.0 & 59.6 & \multirow{5}{*}{$\begin{array}{l}\text { Value not } \\
\text { mentioned }\end{array}$} & \multirow{5}{*}{ Small sample size } \\
\hline & & & & & $\begin{array}{c}\text { ORAI } \geq 9 \\
\text { T-score } \leq-2.5\end{array}$ & 65.6 & 78.9 & & \\
\hline & & & & & $\begin{array}{c}\text { OST }<2 \\
\text { T-score } \leq-2.5\end{array}$ & 75.4 & 75.0 & & \\
\hline & & & & & $\begin{array}{c}\text { SCORE } \geq 6 \\
\text { T-score } \leq-2.5\end{array}$ & 83.6 & 53.9 & & \\
\hline & & & & & $\begin{array}{c}\text { Weight Criterion < } \\
70 \mathrm{~kg} \\
\text { T-score } \leq-2.5\end{array}$ & 68.9 & 69.2 & & \\
\hline \multirow{6}{*}{$\begin{array}{l}\text { Zimering et al. (2007) } \\
\text { [55] }\end{array}$} & \multirow{6}{*}{$\begin{array}{c}\text { To compare a novel } \\
\text { osteoporosis screening tool } \\
\text { with OST in predicting low } \\
\text { BMD }\end{array}$} & \multirow{6}{*}{$\begin{array}{c}\text { Development phase: } \\
\text { Caucasian men (mean age: } \\
68.4 \pm 10.2 \text { years) } \\
\text { Validation phase: } \\
\text { Caucasian men (mean age: } \\
68.4 \pm 10.2 \text { years) } \\
\text { African American men } \\
\text { (mean age: } 60.9 \pm 13 \text { years) }\end{array}$} & \multirow{6}{*}{$\begin{array}{c}\text { Development: } \\
639 \text { Caucasian men } \\
\text { Validation: } \\
\text { 197 Caucasian men } \\
134 \text { African American }\end{array}$} & \multirow{6}{*}{$\begin{array}{c}\text { DXA } \\
\text { Hologic QDR 4500 SL } \\
\text { machine (Waltham, MA, } \\
\text { USA) } \\
\text { BMD at FN, TH and LS }\end{array}$} & $\begin{array}{c}\text { Caucasian } \\
\mathrm{M}_{\text {score }}(\text { cutoff }=9) \\
\text { FN T-score } \leq-2.5\end{array}$ & 88 & 57 & 0.84 & \multirow{6}{*}{$\begin{array}{l}\mathrm{M}_{\text {score is the first }} \\
\text { validated risk } \\
\text { assessment tool } \\
\text { developed in men }\end{array}$} \\
\hline & & & & & $\begin{array}{c}\text { OST (cutoff }=4 \text { ) } \\
\text { FN T-score } \leq-2.5\end{array}$ & 85 & 51 & 0.81 & \\
\hline & & & & & $\begin{array}{c}\text { M score age-weight } \\
\text { (cutoff }=9 \text { ) } \\
\text { FN T-score } \leq-2.5\end{array}$ & 85 & 58 & 0.81 & \\
\hline & & & & & $\begin{array}{c}\text { African American } \\
\mathrm{M}_{\text {score }}=9 \\
\text { FN T-score } \leq-2.5\end{array}$ & NT & NT & NT & \\
\hline & & & & & $\begin{array}{c}\text { OST (cutoff }=4) \\
\text { FN T-score } \leq-2.5\end{array}$ & 100 & 72 & 0.99 & \\
\hline & & & & & $\begin{array}{l}\mathrm{M}_{\text {score age-weight }} \\
\quad \text { (cutoff }=9) \\
\text { FN T-score } \leq-2.5\end{array}$ & 100 & 73 & 0.99 & \\
\hline \multirow{6}{*}{$\begin{array}{c}\text { Jiang et al. (2016) } \\
\text { [52] }\end{array}$} & \multirow{6}{*}{$\begin{array}{l}\text { To compare the } \\
\text { performance of screening } \\
\text { tools with BMI alone in } \\
\text { identifying early } \\
\text { postmenopausal women } \\
\text { with OP }\end{array}$} & \multirow{6}{*}{$\begin{array}{l}\text { Postmenopausal women } \\
\text { (mean age: } 57 \pm 4.2 \text { years) }\end{array}$} & \multirow{6}{*}{445} & \multirow{6}{*}{ DXA } & BMI $<28$ & 95 & 38 & 0.73 & \multirow{6}{*}{$\begin{array}{l}\text { Small sample size } \\
\text { Low statistical power } \\
\text { of detecting the } \\
\text { difference in AUCs }\end{array}$} \\
\hline & & & & & $\begin{array}{c}\text { OST }<2 \\
\text { T-score } \leq-2.5\end{array}$ & 79 & 56 & 0.73 & \\
\hline & & & & & $\begin{array}{c}\text { ORAI } \geq 9 \\
\text { T-score } \leq-2.5\end{array}$ & 74 & 60 & 0.69 & \\
\hline & & & & & $\begin{array}{c}\text { SCORE } \geq 6 \\
\text { T-score } \leq-2.5\end{array}$ & 92 & 34 & 0.75 & \\
\hline & & & & & USPSTF $\geq 9.3 \%$ & 24 & 83 & 0.62 & \\
\hline & & & & & $\mathrm{RF} \geq 1$ risk factors & 66 & 62 & 0.64 & \\
\hline
\end{tabular}


Table 2. Cont.

\begin{tabular}{|c|c|c|c|c|c|c|c|c|c|}
\hline \multirow{4}{*}{$\begin{array}{l}\text { Pecina et al. (2016) } \\
\text { [53] }\end{array}$} & \multirow{4}{*}{$\begin{array}{l}\text { To compare the } \\
\text { effectiveness of risk tools to } \\
\text { predict OP in women aged } \\
50-64\end{array}$} & \multirow{4}{*}{$\begin{array}{l}\text { Retrospective data of women } \\
\text { (mean age: } 56.6 \pm 3.4 \text { ) who } \\
\text { underwent DXA scan in a } \\
\text { clinic }\end{array}$} & \multirow{4}{*}{290} & \multirow{4}{*}{$\begin{array}{c}\text { DXA } \\
\text { BMD at hip/LS }\end{array}$} & $\begin{array}{c}\text { USPSTF FRAX } \geq \\
9.3 \%\end{array}$ & 36 & 73 & 0.55 & \\
\hline & & & & & SCORE $\geq 6$ & 74 & 42 & 0.58 & \\
\hline & & & & & OST $<2$ & 56 & 69 & 0.63 & \\
\hline & & & & & ORAI $\geq 9$ & 52 & 67 & 0.60 & \\
\hline \multirow{2}{*}{$\begin{array}{l}\text { Hawker et al. (2012) } \\
\text { [56] }\end{array}$} & \multirow{2}{*}{$\begin{array}{l}\text { To develop a screening tool } \\
\text { to guide bone density } \\
\text { testing in healthy mid-life } \\
\text { women }\end{array}$} & \multirow{2}{*}{$\begin{array}{l}\text { Healthy women (age range } \\
40-60) \text { receiving their first } \\
\text { BMD in an urban teaching } \\
\text { hospital } \\
\end{array}$} & \multirow[t]{2}{*}{944} & \multirow{2}{*}{$\begin{array}{c}\text { DXA } \\
\text { Lunar Prodigy (GE Healthcare, } \\
\text { Madison WI, USA) } \\
\text { BMD at FN, TH and LS } \\
\end{array}$} & $\begin{array}{c}\text { New tool } \\
\text { T-score } \leq-2.0\end{array}$ & 93 & 36 & 0.75 & \multirow[t]{2}{*}{$\begin{array}{l}\text { Only Caucasian } \\
\text { population is involved }\end{array}$} \\
\hline & & & & & $\begin{array}{c}\text { OST } \leq 1 \\
\text { T-score } \leq-2.0\end{array}$ & 47 & $\begin{array}{l}\text { Value not } \\
\text { mentioned }\end{array}$ & 0.69 & \\
\hline \multirow{5}{*}{ 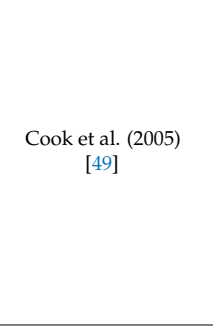 } & \multirow{5}{*}{$\begin{array}{l}\text { To assess the performance } \\
\text { of various osteoporosis } \\
\text { screening tools and } \\
\text { quantitative ultrasound in } \\
\text { relation to DXA scan }\end{array}$} & \multirow{5}{*}{$\begin{array}{l}\text { Postmenopausal women (age } \\
\text { range: } 29-87 \text { years) recruited } \\
\text { from DXA scanning clinics }\end{array}$} & \multirow{5}{*}{208} & \multirow{5}{*}{$\begin{array}{c}\text { DXA } \\
\text { Hologic QDR 4500 C (Hologic Inc., } \\
\text { Bedford, MA, USA) } \\
\text { BMD at LS and PF }\end{array}$} & $\begin{array}{c}\text { OST }<-1 \\
\text { T-score } \leq-2.5\end{array}$ & 0.52 & 0.82 & 0.716 & \\
\hline & & & & & $\begin{array}{c}\text { SCORE } \\
\text { T-score } \leq-2.5\end{array}$ & 0.5 & 0.83 & 0.720 & \\
\hline & & & & & $\begin{array}{c}\text { ORAI } \\
\text { T-score } \leq-2.5\end{array}$ & 0.43 & 0.86 & 0.664 & \\
\hline & & & & & $\begin{array}{c}\text { QUS } \\
\text { BUA calcaneus } \\
\text { T-score } \leq-2.5 \\
\end{array}$ & 0.56 & 0.92 & 0.766 & \\
\hline & & & & & $\begin{array}{l}\text { VOS calcaneus } \\
\text { T-score } \leq-2.5\end{array}$ & 0.61 & 0.72 & 0.723 & \\
\hline \multirow{2}{*}{$\begin{array}{l}\text { Perez-Castrillon et al. } \\
\qquad \begin{array}{c}(2007) \\
{[46]}\end{array}\end{array}$} & \multirow{2}{*}{$\begin{array}{l}\text { To identify if the } \\
\text { combination of OST and } \\
\text { calcaneal DXA improves } \\
\text { the diagnosis of OP }\end{array}$} & \multirow{2}{*}{$\begin{array}{l}\text { Males with a mean age of } 47 \\
\pm 13 \text { years and females with } \\
\text { mean age of f6 } \pm 8 \text { years } \\
\text { recruited from two } \\
\text { university hospitals }\end{array}$} & \multirow[t]{2}{*}{$\begin{array}{l}67 \text { males } \\
94 \text { females }\end{array}$} & \multirow{2}{*}{$\begin{array}{c}\text { DXA: } \\
\text { Pixi-Lunar, DPXL Lunar (Madison, } \\
\text { WI, USA) and Hologic QDR-4500; } \\
\text { Hologic Inc. (Bedford, MD, USA) } \\
\text { BMD at right calcaneal and hip }\end{array}$} & $\begin{array}{c}\text { Men } \\
\text { OST } \leq 3 \\
\text { T-score }<-2.5 \\
\end{array}$ & 39 & 86 & 0.623 & \multirow[t]{2}{*}{ Small sample size } \\
\hline & & & & & $\begin{array}{c}\text { Women } \\
\text { OST } \leq 2 \\
\text { T-score }<-2.5 \\
\end{array}$ & 94 & 59 & 0.762 & \\
\hline $\begin{array}{l}\text { Richards et al. (2009) } \\
\text { [47] }\end{array}$ & $\begin{array}{c}\text { To evaluate the } \\
\text { performance of OST in } \\
\text { predicting low BMD in } \\
\text { male patients with } \\
\text { rheumatoid arthritis } \\
\end{array}$ & $\begin{array}{l}\text { Males (mean age: } 65.4 \pm 10.5 \\
\text { years) recruited from a } \\
\text { multicenter registry of } \\
\text { rheumatoid arthritis }\end{array}$ & 795 & $\begin{array}{c}\text { DXA } \\
\text { Hologic Inc. (Bedford, MA, USA) } \\
\text { BMD at Femur and LS }\end{array}$ & OST $\leq 4$ & 64 & 54 & $\begin{array}{c}\text { Not } \\
\text { mentioned }\end{array}$ & $\begin{array}{l}\text { Low lean body mass in } \\
\text { RA could limit the } \\
\text { utility of the STT in } \\
\text { this population }\end{array}$ \\
\hline
\end{tabular}

Abbreviation: AP, anteroposterior; AUC, area under curve; BMD, bone mineral density; LS, lumbar spine; FN, femoral neck; TH, total hip; NT, not tested; OP, osteoporosis; PF, proximal

femur; QUS, quantitative ultrasound; RF, Risk Factor-Based Approach; USPSTF, the U.S. Preventive Services Task Force; WBC, Weight-based Criterion. 


\section{OST for Fracture Prediction}

Fragility fracture is one of the most common complications of osteoporosis. Although OST was developed to identify individuals with low BMD, its ability to predict fracture risk was assessed in several studies $[54,58,59]$. Yang et al. (2013) reported that OSTA (cutoff $<-1$ ) performed well in determining new vertebral fractures among postmenopausal Chinese women (sensitivity $=81.7 \%$; specificity $=66 \%$; AUC $=0.812$ ) [59].

In comparison with other fracture prediction tools, the performance of OST was weaker than DXA [58] and FRAX [58] in Chinese men and the Singh index in patients with type 2 diabetes mellitus [60]. However, a study showed that USPSTF (FRAX) (sensitivity $=25.8 \%$; specificity $=83.3 \%$; AUC $=0.56$ ) was not better than OST in fracture prediction (sensitivity $=39.8 \%$; specificity $=60.7 \%$; AUC $=0.52$ ) among non-Asian postmenopausal women [54].

A summary of the literature on the performance of OST to predict fracture risk is listed in Table 3. 
Table 3. Performance of OST to predict fracture risk.

\begin{tabular}{|c|c|c|c|c|c|c|c|c|c|}
\hline Study & Objective & Subject Description & $\begin{array}{c}\text { Number of Subjects } \\
\text { Recruited }\end{array}$ & Methods & Cutoff & $\begin{array}{c}\text { Sensitivity } \\
(\%)\end{array}$ & $\begin{array}{c}\text { Specificity } \\
(\%)\end{array}$ & AUC & Remarks \\
\hline $\begin{array}{l}\text { Yang et al. (2013) } \\
{[53]}\end{array}$ & $\begin{array}{c}\text { To validate the performance of } \\
\text { OSTA in determining vertebral } \\
\text { fracture among postmenopausal } \\
\text { women in China }\end{array}$ & $\begin{array}{c}\text { Postmenopausal women (average age: } 62 \\
\text { years) recruited from OP clinic in Beijing, } \\
\text { China }\end{array}$ & 1201 & $\begin{array}{c}\text { DXA Hologic, Inc. (Bedford, MA, } \\
\text { USA) } \\
\text { BMD at LS, FN and TH }\end{array}$ & $\begin{array}{l}\text { OSTA }<-1 \\
\text { and fracture }\end{array}$ & 81.7 & 66 & 0.812 & $\begin{array}{l}\text { All subjects are } \\
\text { recruited from one } \\
\text { single OP centre }\end{array}$ \\
\hline \multirow{3}{*}{$\begin{array}{l}\text { Crandall et al. (2014) } \\
\text { [54] }\end{array}$} & \multirow{3}{*}{$\begin{array}{l}\text { To compare the performance of } \\
\text { USPSTFS, OST and SCORE in } \\
\text { predicting fracture risk among } \\
\text { postmenopausal women }\end{array}$} & \multirow{3}{*}{$\begin{array}{l}\text { Postmenopausal women aged 50-64 years } \\
\text { who participated in Women's Health } \\
\text { Initiative Observational Study and } \\
\text { Clinical Trials }\end{array}$} & \multirow{3}{*}{62,492} & \multirow{3}{*}{$\begin{array}{c}\text { DXA } \\
\text { Hologic QDR2000 or QDR4500 } \\
\text { (Bedford, MA, USA) } \\
\text { BMD at hip or LS }\end{array}$} & $\begin{array}{l}\text { USPSTF(FRAX) } \\
\quad \geq 9.3 \%\end{array}$ & 25.8 & 83.3 & 0.56 & \multirow{8}{*}{$\begin{array}{c}\text { Subjects from a single } \\
\text { centre } \\
\text { Two different groups of } \\
\text { population were } \\
\text { involved } \\
\text { OSTA less effective in } \\
\text { predicting risk }\end{array}$} \\
\hline & & & & & SCORE $>7$ & 38.6 & 65.8 & 0.53 & \\
\hline & & & & & OST $<2$ & 39.8 & 60.7 & 0.52 & \\
\hline \multirow{5}{*}{$\begin{array}{l}\text { Lin et al. (2016) } \\
{[55]}\end{array}$} & \multirow{5}{*}{$\begin{array}{l}\text { To validate the use of three tools } \\
\text { in predicting new osteoporotic } \\
\text { fractures in older Chinese men }\end{array}$} & \multirow{5}{*}{ Han Chinese men aged 50 and above } & \multirow{5}{*}{496} & \multirow{5}{*}{$\begin{array}{c}\text { DXA } \\
\text { Discovery Wi, QDR, Hologic } \\
\text { (Waltham, MA, USA) } \\
\text { BMD at FN, LS and TH }\end{array}$} & TH T-score $<-1.4$ & 67.57 & 65.45 & 0.711 & \\
\hline & & & & & FN T-score $<-2.5$ & 42.34 & 89.87 & 0.706 & \\
\hline & & & & & LS T-score $<-1.6$ & 52.25 & 77.14 & 0.706 & \\
\hline & & & & & FRAX $>2.9$ & 81.98 & 62.08 & 0.738 & \\
\hline & & & & & OSTA $<-1.2$ & 53.15 & 76.88 & 0.661 & \\
\hline \multirow{6}{*}{$\begin{array}{l}\text { Liu et al. (2017) } \\
\quad[56]\end{array}$} & \multirow{6}{*}{$\begin{array}{l}\text { To evaluate the performance of } \\
\text { Singh score and OSTA in } \\
\text { predicting hip fracture in } \\
\text { patients with type } 2 \text { diabetes } \\
\text { mellitus }\end{array}$} & \multirow{6}{*}{$\begin{array}{l}\text { Postmenopausal women with } 87 \text { of them } \\
\text { (age range: } 56-86 \text { years) had a hip fracture }\end{array}$} & \multirow{6}{*}{261} & \multirow{6}{*}{$\begin{array}{c}\text { DXA } \\
\text { Discovery, Hologic, Inc. } \\
\text { (Bedford, MA, USA) } \\
\text { BMD at hip and LS } \\
\text { Retrospective Singh score: } \\
\text { Standard digital anteroposterior } \\
\text { radiographs }\end{array}$} & LS T-score $<-1.85$ & 60.9 & 77 & 0.747 & \multirow{6}{*}{ Small sample size } \\
\hline & & & & & TH T-score $<-2.45$ & 52.9 & 71.8 & 0.699 & \\
\hline & & & & & FN T-score $<-2.05$ & 74.7 & 47.1 & 0.659 & \\
\hline & & & & & $\begin{array}{l}\text { Femoral trochanter } \\
\text { T-score }<-2.25\end{array}$ & 50.6 & 69.5 & 0.631 & \\
\hline & & & & & OSTA $<-2.5$ & 44.8 & 73.8 & 0.534 & \\
\hline & & & & & $\begin{array}{l}\text { Singh index }<2.5 \\
\text { OSTA and Singh }\end{array}$ & $\begin{array}{c}42.5 \\
\text { Value not } \\
\text { mentioned }\end{array}$ & $\begin{array}{c}88.2 \\
\text { Value not } \\
\text { mentioned }\end{array}$ & $\begin{array}{l}0.636 \\
0.795\end{array}$ & \\
\hline
\end{tabular}

AUC, area under curve; BMD, bone mineral density; LS, lumbar spine; FN, femoral neck; TH, total hip; OP, osteoporosis; USPSTF, the U.S. Preventive Services Task Force. 


\section{Conclusions}

The performance of OST in predicting osteoporosis has been tested in various Asian and non-Asian populations. It demonstrates good predictive values in terms of sensitivity, specificity, and AUC when BMD is used as the reference. Some modifications in the OST cutoff should be made and tested to optimize its performance prior to its deployment, since the performance may vary according to age, sex, ethnicities, and the site of BMD measurement. Validation studies are necessary before including OST in the national guideline for osteoporosis screening. In most studies, OST demonstrated a high sensitivity and low specificity, which is typical for a screening test. In other words, OST might direct some individuals with normal bone health for an unnecessary DXA scan. At the same time, the number of potential patients subjected to a DXA scan is maximized, allowing the early detection and treatment of osteoporosis. This will reduce the complications and burdens of the disease. Thus, we argue that the benefits of implementing OST will outweigh its cost. As a conclusion, OST is a useful osteoporosis screening tool in prioritizing high-risk individuals for a DXA scan. It enables early disease detection, optimizes the use of the diagnostic facility, and therefore reduces the disease burden of osteoporosis.

Author Contributions: S.S. performed the literature search and drafted the manuscript. K.-Y.C. and S.I.-N. critically reviewed and expanded the text. All authors have approved the final, submitted manuscript.

Funding: We thank Universiti Kebangsaan Malaysia for funding this study via grants GUP-2017-060 and AP-2017-009/1.

Conflicts of Interest: The authors declare no conflict of interest.

\section{References}

1. Tao, B.; Liu, J.M.; Li, X.Y.; Wang, J.G.; Wang, W.Q.; Ning, G. An assessment of the use of quantitative ultrasound and the osteoporosis self-assessment tool for asians in determining the risk of nonvertebral fracture in postmenopausal chinese women. J. Bone Miner. Metab. 2008, 26, 60-65. [CrossRef] [PubMed]

2. Cheung, C.-L.; Ang, S.B.; Chadha, M.; Chow, E.S.-L.; Chung, Y.-S.; Hew, F.L.; Jaisamrarn, U.; Ng, H.; Takeuchi, Y.; Wu, C.-H. An updated hip fracture projection in asia: The asian federation of osteoporosis societies study. Osteoporos. Sarcopenia 2018, 4, 16-21. [CrossRef]

3. Oden, A.; McCloskey, E.V.; Kanis, J.A.; Harvey, N.C.; Johansson, H. Burden of high fracture probability worldwide: Secular increases 2010-2040. Osteoporos. Int. 2015, 26, 2243-2248. [CrossRef] [PubMed]

4. Zha, X.Y.; Hu, Y.; Pang, X.N.; Chang, G.L.; Li, L. Diagnostic value of osteoporosis self-assessment tool for asians (osta) and quantitative bone ultrasound (qus) in detecting high-risk populations for osteoporosis among elderly chinese men. J. Bone Miner. Metab. 2015, 33, 230-238. [CrossRef] [PubMed]

5. Mohd-Tahir, N.A.; Li, S.C. Economic burden of osteoporosis-related hip fracture in Asia: A systematic review. Osteoporos. Int. 2017, 28, 2035-2044. [CrossRef] [PubMed]

6. World Health Organization. Assessment of Fracture Risk and Its Application to Screening for Postmenopausal Osteoporosis: Report of a Who Study Group [Meeting Held in Rome from 22 to 25 June 1992]; World Health Organization: Geneva, Switzerland, 1994.

7. Chin, K.Y.; Ima-Nirwana, S. Calcaneal quantitative ultrasound as a determinant of bone health status: What properties of bone does it reflect? Int. J. Med. Sci. 2013, 10, 1778-1783. [CrossRef] [PubMed]

8. Cherian, K.E.; Kapoor, N.; Shetty, S.; Naik, D.; Thomas, N.; Paul, T.V. Evaluation of different screening tools for predicting femoral neck osteoporosis in rural south indian postmenopausal women. J. Clin. Densitom. 2018, 21, 119-124. [CrossRef] [PubMed]

9. Rubin, K.H.; Friis-Holmberg, T.; Hermann, A.P.; Abrahamsen, B.; Brixen, K. Risk assessment tools to identify women with increased risk of osteoporotic fracture: Complexity or simplicity? A systematic review. J. Bone Miner. Res. 2013, 28, 1701-1717. [CrossRef] [PubMed]

10. Richy, F.; Gourlay, M.; Ross, P.; Sen, S.; Radican, L.; De Ceulaer, F.; Ben Sedrine, W.; Ethgen, O.; Bruyère, O.; Reginster, J.-Y. Validation and comparative evaluation of the osteoporosis self-assessment tool (ost) in a caucasian population from belgium. QJM 2004, 97, 39-46. [CrossRef] [PubMed] 
11. Koh, L.K.; Sedrine, W.B.; Torralba, T.P.; Kung, A.; Fujiwara, S.; Chan, S.P.; Huang, Q.R.; Rajatanavin, R.; Tsai, K.S.; Park, H.M.; et al. A simple tool to identify asian women at increased risk of osteoporosis. Osteoporos. Int. 2001, 12, 699-705. [CrossRef] [PubMed]

12. Kung, A.W.; Ho, A.Y.; Ross, P.D.; Reginster, J.Y. Development of a clinical assessment tool in identifying asian men with low bone mineral density and comparison of its usefulness to quantitative bone ultrasound. Osteoporos. Int. 2005, 16, 849-855. [CrossRef] [PubMed]

13. Chin, K.Y. A review on the performance of osteoporosis self-assessment tool for asians in determining osteoporosis and fracture risk. Postgrad. Med. 2017, 129, 734-746. [CrossRef] [PubMed]

14. Black, D. Sofsurf: A simple, useful risk factor system can identify the large majority of women with osteoporosis. Bone 1998, 23, S605.

15. Lydick, E.; Turpin, J.; Cook, K.; Stine, R.; Melton, M.; Byrnes, C. Development and validation of a simple questionnaire to facilitate identification of women with low bone density. J. Bone Miner. Res. 1996, 11, S368.

16. Huang, J.Y.; Song, W.Z.; Zeng, H.R.; Huang, M.; Wen, Q.F. Performance of the osteoporosis self-assessment tool for asians (osta) in screening osteoporosis among middle-aged and old women in the chengdu region of china. J. Clin. Densitom. 2015, 18, 539-545. [CrossRef] [PubMed]

17. Park, H.M.; Sedrine, W.B.; Reginster, J.Y.; Ross, P.D. Korean experience with the osta risk index for osteoporosis: A validation study. J. Clin. Densitom. 2003, 6, 247-250. [CrossRef]

18. Geater, S.; Leelawattana, R.; Geater, A. Validation of the osta index for discriminating between high and low probability of femoral neck and lumbar spine osteoporosis among thai postmenopausal women. J. Med. Assoc. Thai. 2004, 87, 1286-1292. [PubMed]

19. Chaovisitsaree, S.; Namwongprom, S.N.; Morakote, N.; Suntornlimsiri, N.; Piyamongkol, W. Comparison of osteoporosis self assessment tool for asian (osta) and standard assessment in menopause clinic, chiang mai. J. Med. Assoc. Thai. 2007, 90, 420-425. [PubMed]

20. Harvey, N.; Dennison, E.; Cooper, C. Osteoporosis: Impact on health and economics. Nat. Rev. Rheumatol. 2010, 6, 99-105. [CrossRef] [PubMed]

21. Yang, Y.; Li, D.; Fei, Q.; Wang, B.Q.; Tang, H.; Li, J.J.; Meng, Q.; Zhao, F. Self-assessment tool to identify primary osteoporosis in chinese elderly males. Int. J. Gerontol. 2015, 9, 71-76. [CrossRef]

22. Oh, S.M.; Song, B.M.; Nam, B.-H.; Rhee, Y.; Moon, S.-H.; Kim, D.Y.; Kang, D.R.; Kim, H.C. Development and validation of osteoporosis risk-assessment model for korean men. Yonsei Med. J. 2016, 57, 187-196. [CrossRef] [PubMed]

23. Huang, J.-Y.; Song, W.-Z.; Huang, M. Effectiveness of osteoporosis self-assessment tool for asians in screening for osteoporosis in healthy males over 40 years old in china. J. Clin. Densitom. 2017, 20, 153-159. [CrossRef] [PubMed]

24. Bhat, K.A.; Kakaji, M.; Awasthi, A.; Kumar, K.; Mishra, K.; Shukla, M.; Gupta, S.K. Utility of osteoporosis self-assessment tool as a screening tool for predicting osteoporosis in indian men. J. Clin. Densitom. 2017, 20, 160-163. [CrossRef] [PubMed]

25. Chang, S.F.; Yang, R.S. Optimal analysis to discriminate males' osteoporosis with simple physiological indicators: A cutoff point study. Am. J. Mens Health 2016, 10, 487-494. [CrossRef] [PubMed]

26. Trimpou, P.; Bosaeus, I.; Bengtsson, B.-Å.; Landin-Wilhelmsen, K. High correlation between quantitative ultrasound and dxa during 7 years of follow-up. Eur. J. Radiol. 2010, 73, 360-364. [CrossRef] [PubMed]

27. Chen, S.J.; Chen, Y.J.; Cheng, C.H.; Hwang, H.F.; Chen, C.Y.; Lin, M.R. Comparisons of different screening tools for identifying fracture/osteoporosis risk among community-dwelling older people. Medicine (Baltimore) 2016, 95, e3415. [CrossRef] [PubMed]

28. Kung, A.W.; Ho, A.Y.; Sedrine, W.B.; Reginster, J.Y.; Ross, P.D. Comparison of a simple clinical risk index and quantitative bone ultrasound for identifying women at increased risk of osteoporosis. Osteoporos. Int. 2003, 14, 716-721. [CrossRef] [PubMed]

29. Chin, K.Y.; Low, N.Y.; Kamaruddin, A.A.A.; Dewiputri, W.I.; Soelaiman, I.N. Agreement between calcaneal quantitative ultrasound and osteoporosis self-assessment tool for asians in identifying individuals at risk of osteoporosis. Ther. Clin. Risk Manag. 2017, 13, 1333-1341. [CrossRef] [PubMed]

30. Chan, S.P.; Teo, C.C.; Ng, S.A.; Goh, N.; Tan, C.; Deurenberg-Yap, M. Validation of various osteoporosis risk indices in elderly chinese females in singapore. Osteoporos. Int. 2006, 17, 1182-1188. [CrossRef] [PubMed] 
31. Panichyawat, N.; Tanmahasamut, P. Comparison of osta index and kkos scoring system for prediction of osteoporosis in postmenopausal women who attended siriraj menopause clinic. J. Med. Assoc. Thail. 2012, 95, 1365-1371.

32. Lin, J.; Yang, Y.; Zhang, X.; Ma, Z.; Wu, H.; Li, Y.; Yang, X.; Fei, Q.; Guo, A. Bfh-ostm, a new predictive screening tool for identifying osteoporosis in elderly han chinese males. Clin. Interv. Aging 2017, 12, 1167-1174. [CrossRef] [PubMed]

33. Ma, Z.; Yang, Y.; Lin, J.; Zhang, X.; Meng, Q.; Wang, B.; Fei, Q. Bfh-ost, a new predictive screening tool for identifying osteoporosis in postmenopausal han chinese women. Clin. Interv. Aging 2016, 11, 1051-1059. [PubMed]

34. Satyaraddi, A.; Shetty, S.; Kapoor, N.; Cherian, K.E.; Naik, D.; Thomas, N.; Paul, T.V. Performance of risk assessment tools for predicting osteoporosis in south indian rural elderly men. Arch. Osteoporos. 2017, 12, 35. [CrossRef] [PubMed]

35. Chen, J.-H.; Chen, Y.-C.; Tsai, M.-K.; Chiou, J.-M.; Lee, W.-C.; Tsao, C.-K.; Tsai, K.-S.; Chie, W.-C. Predicting the risk of osteopenia for women aged 40-55 years. J. Formos. Med. Assoc. 2017, 116, 888-896. [CrossRef] [PubMed]

36. Oh, S.M.; Nam, B.H.; Rhee, Y.; Moon, S.H.; Kim, D.Y.; Kang, D.R.; Kim, H.C. Development and validation of osteoporosis risk-assessment model for korean postmenopausal women. J. Bone Miner. Metab. 2013, 31, 423-432. [CrossRef] [PubMed]

37. Pei Shan, L.; Fee Bee, O.; Seri Suniza, S.; Adeeb, N. Developing a malaysian osteoporosis screening tool (most) for early osteoporosis detection in malaysian women. Sex. Reprod. Healthc. 2011, 2, 77-82. [CrossRef] [PubMed]

38. Cadarette, S.M.; McIsaac, W.J.; Hawker, G.A.; Jaakkimainen, L.; Culbert, A.; Zarifa, G.; Ola, E.; Jaglal, S.B. The validity of decision rules for selecting women with primary osteoporosis for bone mineral density testing. Osteoporos. In.t 2004, 15, 361-366.

39. Gourlay, M.L.; Miller, W.C.; Richy, F.; Garrett, J.M.; Hanson, L.C.; Reginster, J.Y. Performance of osteoporosis risk assessment tools in postmenopausal women aged 45-64 years. Osteoporos. Int. 2005, 16, 921-927. [CrossRef] [PubMed]

40. Adler, R.A.; Tran, M.T.; Petkov, V.I. Performance of the osteoporosis self-assessment screening tool for osteoporosis in american men. Mayo. Clin. Proc. 2003, 78, 723-727. [CrossRef] [PubMed]

41. Ghazi, M.; Mounach, A.; Nouijai, A.; Ghozlani, I.; Bennani, L.; Achemlal, L.; Bezza, A.; El Maghraoui, A. Performance of the osteoporosis risk assessment tool in moroccan men. Clin. Rheumatol. 2007, 26, 2037-2041. [CrossRef] [PubMed]

42. Sinnott, B.; Kukreja, S.; Barengolts, E. Utility of screening tools for the prediction of low bone mass in african american men. Osteoporos. Int. 2006, 17, 684-692. [CrossRef] [PubMed]

43. Lynn, H.S.; Woo, J.; Leung, P.C.; Barrett-Connor, E.L.; Nevitt, M.C.; Cauley, J.A.; Adler, R.A.; Orwoll, E.S. An evaluation of osteoporosis screening tools for the osteoporotic fractures in men (mros) study. Osteoporos. Int. 2008, 19, 1087-1092. [CrossRef] [PubMed]

44. Machado, P.; Coutinho, M.; Da Silva, J. Selecting men for bone densitometry: Performance of osteoporosis risk assessment tools in portuguese men. Osteoporos Int 2010, 21, 977-983. [CrossRef] [PubMed]

45. Steuart Richards, J.; Lazzari, A.A.; Teves Qualler, D.A.; Desale, S.; Howard, R.; Kerr, G.S. Validation of the osteoporosis self-assessment tool in us male veterans. J. Clin. Densitom. 2014, 17, 32-37. [CrossRef] [PubMed]

46. Pérez-Castrillón, J.L.; Sagredo, M.G.; Conde, R.; del Pino-Montes, J.; de Luis, D. Ost risk index and calcaneus bone densitometry in osteoporosis diagnosis. J. Clin Densitom 2007, 10, 404-407. [CrossRef] [PubMed]

47. Richards, J.S.; Peng, J.; Amdur, R.L.; Mikuls, T.R.; Hooker, R.S.; Michaud, K.; Reimold, A.M.; Cannon, G.W.; Caplan, L.; Johnson, D.; et al. Dual-energy x-ray absorptiometry and evaluation of the osteoporosis self-assessment tool in men with rheumatoid arthritis. J. Clin. Densitom. 2009, 12, 434-440. [CrossRef] [PubMed]

48. Wallace, L.S.; Ballard, J.E.; Holiday, D.; Turner, L.W.; Keenum, A.J.; Pearman, C.M. Evaluation of decision rules for identifying low bone density in postmenopausal african-american women. J. Natl. Med. Assoc. 2004, 96, 290-296. [PubMed]

49. Cook, R.B.; Collins, D.; Tucker, J.; Zioupos, P. Comparison of questionnaire and quantitative ultrasound techniques as screening tools for dxa. Osteoporos. Int. 2005, 16, 1565-1575. [CrossRef] [PubMed] 
50. Crandall, C.J.; Larson, J.; Gourlay, M.L.; Donaldson, M.G.; LaCroix, A.; Cauley, J.A.; Wactawski-Wende, J.; Gass, M.L.; Robbins, J.A.; Watts, N.B.; et al. Osteoporosis screening in postmenopausal women 50 to 64 years old: Comparison of us preventive services task force strategy and two traditional strategies in the women's health initiative. J. Bone Miner. Res. 2014, 29, 1661-1666. [CrossRef] [PubMed]

51. Geusens, P.; Hochberg, M.C.; van der Voort, D.J.M.; Pols, H.; van der Klift, M.; Siris, E.; Melton, M.E.; Turpin, J.; Byrnes, C.; Ross, P.D. Performance of risk indices for identifying low bone density in postmenopausal women. Mayo. Clin. Proc. 2002, 77, 629-637. [CrossRef] [PubMed]

52. Jiang, X.; Good, L.E.; Spinka, R.; Schnatz, P.F. Osteoporosis screening in postmenopausal women aged 50-64 years: Bmi alone compared with current screening tools. Maturitas 2016, 83, 59-64. [CrossRef] [PubMed]

53. Pecina, J.L.; Romanovsky, L.; Merry, S.P.; Kennel, K.A.; Thacher, T.D. Comparison of clinical risk tools for predicting osteoporosis in women ages 50-64. J. Am. Board Fam. Med. 2016, 29, 233-239. [CrossRef] [PubMed]

54. Crandall, C.J.; Larson, J.C.; Watts, N.B.; Gourlay, M.L.; Donaldson, M.G.; LaCroix, A.; Cauley, J.A.; Wactawski-Wende, J.; Gass, M.L.; Robbins, J.A.; et al. Comparison of fracture risk prediction by the us preventive services task force strategy and two alternative strategies in women 50-64 years old in the women's health initiative. J. Clin. Endocrino.l Metab. 2014, 99, 4514-4522. [CrossRef] [PubMed]

55. Zimering, M.B.; Shin, J.J.; Shah, J.; Wininger, E.; Engelhart, C. Validation of a novel risk estimation tool for predicting low bone density in caucasian and african american men veterans. J. Clin. Densitom. 2007, 10, 289-297. [CrossRef] [PubMed]

56. Hawker, G.; Mendel, A.; Lam, M.A.; Akhavan, P.S.; Cancino-Romero, J.; Waugh, E.; Jamal, S.; Mian, S.; Jaglal, S. A clinical decision rule to enhance targeted bone mineral density testing in healthy mid-life women. Osteoporos. Int. 2012, 23, 1931-1938. [CrossRef] [PubMed]

57. Richards, J.S.; Lazzari, A.A.; Qualler, D.A.T.; Desale, S.; Howard, R.; Kerr, G.S. Validation of the osteoporosis self-assessment tool in us male veterans. J. Clin. Densitom. 2014, 17, 32-37. [CrossRef] [PubMed]

58. Lin, J.; Yang, Y.; Fei, Q.; Zhang, X.; Ma, Z.; Wang, Q.; Li, J.; Li, D.; Meng, Q.; Wang, B. Validation of three tools for identifying painful new osteoporotic vertebral fractures in older chinese men: Bone mineral density, osteoporosis self-assessment tool for asians, and fracture risk assessment tool. Clin. Interv. Aging 2016, 11, 461-469. [PubMed]

59. Yang, Y.; Wang, B.; Fei, Q.; Meng, Q.; Li, D.; Tang, H.; Li, J.; Su, N. Validation of an osteoporosis self-assessment tool to identify primary osteoporosis and new osteoporotic vertebral fractures in postmenopausal chinese women in beijing. BMC Musculoskelet. Disord. 2013, 14, 271. [CrossRef] [PubMed]

60. Liu, Z.; Gao, H.; Bai, X.; Zhao, L.; Li, Y.; Wang, B. Evaluation of singh index and osteoporosis self-assessment tool for asians as risk assessment tools of hip fracture in patients with type 2 diabetes mellitus. J. Orthop. Surg. Res. 2017, 12, 37. [CrossRef] [PubMed] 